\title{
Sub-surface convection zones in hot massive stars and their observable consequences ${ }^{\star}$
}

\author{
M. Cantiello ${ }^{1}$, N. Langer ${ }^{1,2}$, I. Brott ${ }^{1}$, A. de Koter ${ }^{1,3}$, S. N. Shore ${ }^{4}$, J. S. Vink ${ }^{5}$, \\ A. Voegler ${ }^{1}$, D. J. Lennon ${ }^{6}$, and S.-C. Yoon ${ }^{7}$ \\ 1 Astronomical Institute, Utrecht University, Princetonplein 5, 3584 CC, Utrecht, The Netherlands \\ e-mail:m.cantiello@uu.nl \\ 2 Argelander-Institut für Astronomie der Universität Bonn, Auf dem Hügel 71, 53121 Bonn, Germany \\ 3 Astronomical Institute Anton Pannekoek, University of Amsterdam, Kruislaan 403, 1098 SJ, Amsterdam, The Netherlands \\ 4 Dipartmento di Fisica "Enrico Fermi”, Università di Pisa, via Buonarroti 2, Pisa 56127 and INFN - Sezione di Pisa, Italy \\ 5 Armagh Observatory, College Hill, Armagh, BT61 9DG, Northern Ireland, UK \\ 6 Space Telescope Science Institute, 3700 San Martin Drive, Baltimore, MD 21218, USA \\ 7 Department of Astronomy \& Astrophysics, University of California, Santa Cruz, High Street, Santa Cruz, CA 95064, USA
}

Received 12 January 2009 / Accepted 3 March 2009

\section{ABSTRACT}

\begin{abstract}
Context. We study the convection zones in the outer envelope of hot massive stars which are caused by opacity peaks associated with iron and helium ionization.

Aims. We determine the occurrence and properties of these convection zones as function of the stellar parameters. We then confront our results with observations of $\mathrm{OB}$ stars.

Methods. A stellar evolution code is used to compute a grid of massive star models at different metallicities. In these models, the mixing length theory is used to characterize the envelope convection zones.

Results. We find the iron convection zone $(\mathrm{FeCZ})$ to be more prominent for lower surface gravity, higher luminosity and higher initial metallicity. It is absent for luminosities below about $10^{3.2} L_{\odot}, 10^{3.9} L_{\odot}$, and $10^{4.2} L_{\odot}$ for the Galaxy, LMC and SMC, respectively. We map the strength of the $\mathrm{FeCZ}$ on the Hertzsprung-Russell diagram for three metallicities, and compare this with the occurrence of observational phenomena in $\mathrm{O}$ stars: microturbulence, non-radial pulsations, wind clumping, and line profile variability.

Conclusions. The confirmation of all three trends for the $\mathrm{FeCZ}$ as function of stellar parameters by empirical microturbulent velocities argues for a physical connection between sub-photospheric convective motions and small scale stochastic velocities in the photosphere of O- and B-type stars. We further suggest that clumping in the inner parts of the winds of OB stars could be caused by the same mechanism, and that magnetic fields produced in the FeCZ could appear at the surface of OB stars as diagnosed by discrete absorption components in ultraviolet absorption lines.
\end{abstract}

Key words. convection - stars: early-type - stars: evolution - stars: atmospheres - stars: magnetic fields - stars: oscillations

\section{Introduction}

Massive stars, in a general sense, have convective cores and radiative envelopes (Kippenhahn \& Weigert 1990). The introduction of the so called "iron peak" in stellar opacities (Iglesias et al. 1992) led, however, to the prediction of a small convection zone in the envelope of sufficiently luminous massive main sequence models (Stothers \& Chin 1993). It is often accompanied by an even smaller convection zone which originates from an opacity peak associated with partial helium ionization. These two convection zones comprise almost negligible amount of mass. The reality of the iron opacity bump, as predicted by various groups (e.g., Iglesias et al. 1992; Badnell et al. 2005), is unambiguous. It is most obvious in the field of stellar pulsations. Only the inclusion of this feature allows an agreement of observed and predicted instability regimes in the HR diagram, from the white dwarf regime (e.g. Saio 1993; Charpinet et al. 1997), for main sequence stars (e.g., $\beta$ Cephei stars; see Deng \& Xiong 2001,

\footnotetext{
* Tables A.1 and A.2 are only available in electronic form via http://www . aanda. org
}

and references therein), and up to hot supergiants (Saio et al. 2006).

While the envelope convection zones may, at first glance, be negligible for the internal evolution of hot massive stars, they may cause observable phenomena at the stellar surface. The reason is that the zones are located very close to the photosphere for some mass interval (see below). Here, we will discuss which observed features in hot stars might be produced by these near surface convection zones. In particular, we examine whether a link exists between these convective regions and observable small scale velocity fields at the stellar surface and in the stellar wind, "microturbulence". A similar idea has been used to explain microturbulence in low mass stars (Edmunds 1978), in which deeper envelope convection zones reach the photosphere. While Edmunds (1978) concludes that the same mechanism cannot explain microturbulent velocities in $\mathrm{O}$ and $\mathrm{B}$ stars, the ironpeak induced sub-photospheric convection zones in these stars had not yet been discovered. We demonstrate in this paper that these convection zones may not only cause motions which are observable, but possibly even directly affect the evolution: first, we discuss how photospheric velocity fields may affect the structure of massive star winds by inducing clumping at the base of 
the wind and thereby affecting the stellar mass-loss. And second, we argue that the near surface convection zones may generate magnetic fields which - if they migrate to the surface - further affect the stellar wind mass-loss and, more significantly, the associated stellar angular momentum loss.

We construct grids of massive main sequence star models, for various metallicities, that allow us to predict the occurrence and properties of sub-surface convection zones as function of the stellar parameters (Sect. 3). We then compare the model predictions with observed stellar properties, e.g., empirically derived microturbulent velocities and observations of wind clumping in hot massive stars (Sect. 4).

\section{Method}

Our stellar models are calculated with a hydrodynamic stellar evolution code. This code can calculate the effect of rotation on the stellar structure, rotationally induced chemical mixing, and the transport of angular momentum by magnetic torques (see Petrovic et al. 2005; Yoon et al. 2006, and references therein). Compositional mixing is treated as a diffusive process. The rate of change of a nuclear species of mass fraction $X_{i}$ is calculated as

$$
\left(\frac{\partial X_{i}}{\partial t}\right)=\left(\frac{\partial}{\partial m}\right)\left[\left(4 \pi r^{2} \rho\right)^{2} D\left(\frac{\partial X_{i}}{\partial m}\right)\right]+\left(\frac{\mathrm{d} X_{i}}{\mathrm{~d} t}\right)_{\text {nuc }},
$$

where $D$ is the diffusion coefficient constructed from the sum of individual diffusion coefficients for the range of mixing processes (see Heger et al. 2000, and references therein). The second term on the right hand side is the schematic symbol to stand for all nuclear reactions. The contributions to the diffusion coefficient are convection, semiconvection and thermohaline mixing. For rotating models also the contributions from rotationally induced mixing and magnetic diffusion are computed. The transport of angular momentum is also treated as a diffusive process (Endal \& Sofia 1978; Pinsonneault et al. 1989; Heger et al. 2000).

The Ledoux criterion is used to determine which regions of the star are unstable to convection:

$\nabla_{\mathrm{ad}}-\nabla+\frac{\varphi}{\delta} \nabla_{\mu} \leq 0$

(e.g., Kippenhahn \& Weigert 1990) where $\nabla_{\mathrm{ad}}$ is the adiabatic temperature gradient and $\nabla_{\mu}$ is the gradient in the mean molecular weight. The diffusion coefficient, $D$, in convective regions is approximated with

$D=\frac{1}{3} \alpha H_{\mathrm{P}} v_{\mathrm{c}}$

where $H_{\mathrm{P}}$ is the pressure scale height, $v_{\mathrm{c}}$ is the convective velocity, and $\alpha$ the mixing length parameter. We fix $\alpha=1.5$, which results from evolutionary tracks of the Sun (e.g. Abbett et al. 1997; Ludwig et al. 1999); a sensitivity study of the $\alpha$ dependence of our scenario will be presented in future work. The convective velocity, $v_{\mathrm{c}}$, is calculated using the mixing length theory (Böhm-Vitense 1958) (MLT hereafter) and the convective contribution to the diffusion coefficient becomes:

$D=\frac{1}{3} \alpha^{2 / 3} H_{\mathrm{P}}\left[\frac{c}{\varkappa \rho} g \beta(1-\beta) \nabla_{\mathrm{ad}}\left(\nabla_{\mathrm{rad}}-\nabla_{\mathrm{ad}}\right)\right]^{1 / 3}$,

where $x$ is the opacity, $\rho$ is the density, $\beta$ is the ratio of gas pressure to total pressure, $g$ is the local gravitational acceleration, and $c$ is the speed of light. Here, $\nabla_{\mathrm{rad}}$ and $\nabla_{\mathrm{ad}}$ are the radiative and adiabatic gradients, respectively.

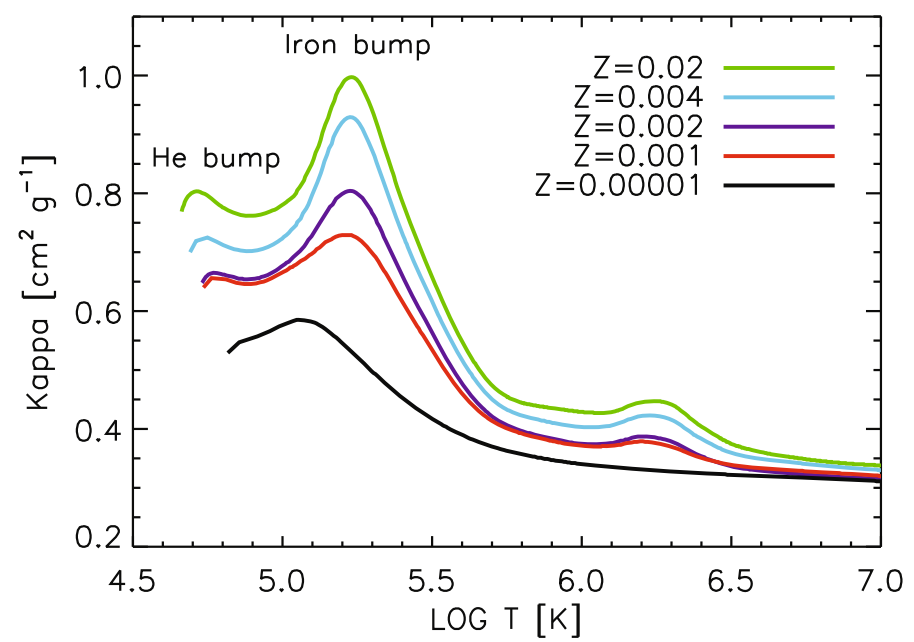

Fig. 1. Opacity in the interior of $60 M_{\odot}$ zero age main sequence stars of various metallicities (see legend) as a function of temperature, from the surface up to a temperature of $10^{7} \mathrm{~K}$. The different colors refer to different metallicities, as shown in the legend.

We use the solar composition proposed by Asplund et al. (2005). The opacities in our code are extracted from the OPAL tables (Iglesias \& Rogers 1996). Figure 1 shows the opacity coefficient as function of temperature in our $60 M_{\odot}$ models for various metallicities. The peaks at $\log T \simeq 4.7$ and $\log T \simeq$ 5.3 are caused by helium and iron, respectively. The peak at $\log T \simeq 6.2-6.3$ is caused by carbon, oxygen and iron.

We use the metallicity dependent mass-loss predictions of Vink et al. (2001).

\subsection{The helium convection zone}

In the very weak helium convection zone, radiative diffusion is the dominant energy transport mechanism, which may have consequences for the development of convection. In fact, in viscous fluids the Ledoux-criterion is not strictly correct, since it ignores any dissipative effect on the evolution of a perturbation. A more accurate criterion can be expressed in terms of the nondimensional Rayleigh number, Ra which for compressible, stratified convection, is

$\mathrm{Ra} \simeq \frac{\left(\nabla-\nabla_{\mathrm{ad}}\right) L^{3} g}{\kappa v}$.

Here $L$ is the thickness of the convective layer, and $\kappa$ and $v$ are, respectively, the thermal diffusivity and the kinematic (molecular) viscosity (e.g, Shore 1992, p. 328).

For convection to develop, Ra must exceed some critical value, $\mathrm{Ra}_{\mathrm{c}}$. The estimate of $\mathrm{Ra}$ in the helium convective region depends on the choice of the viscosity coefficient. For the Spitzer formula (Spitzer 1962), $\mathrm{Ra}>\mathrm{Ra}_{\mathrm{c}}$, and the region can be considered convective. In contrast, for the radiative viscosity (e.g, Kippenhahn \& Weigert 1990, p. 445), $\mathrm{Ra}<\mathrm{Ra}_{\mathrm{c}}$. There is an additional uncertainty in these estimates since the expressions for the radiative transport coefficients in our models are strictly correct only in the diffusion limit. Likewise, the value of the heat capacity $c_{\mathrm{p}}$ can vary by an order of magnitude depending on whether the radiative energy reservoir $a T^{4}$ is coupled to the internal energy of the gas or not. Since the helium convection zone occurs very close to the surface in our models, these additional uncertainties could be relevant. 
Table 1. Properties of the envelope convection zones in our 20 and $60 M_{\odot}$ models of solar metallicity. These are the same models shown in the top panel of Figs. 2 and in 3. The values in the table refer to $t=6.41 \times 10^{6}$ for the $20 M_{\odot}$ model and $t=2.37 \times 10^{6}$ for the $60 M_{\odot}$ model.

\begin{tabular}{lccccccccc}
\hline \hline$M$ & Zone & $\begin{array}{c}H_{\mathrm{P}} \\
M_{\odot}\end{array}$ & $\begin{array}{c}\left\langle v_{\mathrm{c}}\right\rangle \\
R_{\odot}\end{array}$ & $\begin{array}{c}\Delta M_{\text {conv }}{ }^{a} \\
\mathrm{~km} \mathrm{~s}^{-1}\end{array}$ & $\begin{array}{c}\Delta M_{\odot}{ }_{\text {top }}{ }^{b} \\
M_{\odot}\end{array}$ & $N_{\text {cells }^{c}}$ & $\begin{array}{c}\tau_{\text {turn }}{ }^{d} \\
\text { days }\end{array}$ & $\begin{array}{c}\tau_{\text {conv }}{ }^{e} \\
\text { days }\end{array}$ & $\begin{array}{c}\dot{M} \\
M_{\odot} \mathrm{yr}^{-1}\end{array}$ \\
\hline 20 & $\mathrm{He}$ & 0.025 & 0.08 & $7.6 \times 10^{-9}$ & $1.9 \times 10^{-9}$ & $1.8 \times 10^{5}$ & 2.5 & 38 & $7.3 \times 10^{-8}$ \\
20 & $\mathrm{Fe}$ & 0.08 & 2.40 & $3.6 \times 10^{-6}$ & $5.8 \times 10^{-7}$ & $1.8 \times 10^{4}$ & 0.25 & 18250 & $7.3 \times 10^{-8}$ \\
60 & $\mathrm{Fe}$ & 0.24 & 2.25 & $1.6 \times 10^{-5}$ & $9.8 \times 10^{-7}$ & $8.5 \times 10^{3}$ & 0.83 & 1570 & $3.7 \times 10^{-6}$ \\
\hline
\end{tabular}

${ }^{a}$ Mass contained in the convective region. ${ }^{b}$ Mass in the radiative layer between the stellar surface and the upper boundary of the convective zone. ${ }^{c}$ Expected number of convective cells, $N_{\text {cells }}:=\left(R_{\star} / H_{\mathrm{P}}\right)^{2} .{ }^{d}$ Convective turnover time, $\tau_{\text {turn }}:=H_{\mathrm{P}} /\left\langle v_{\mathrm{c}}\right\rangle .{ }^{e}$ Time that a piece of stellar material spends inside a convective region, $\tau_{\text {conv }}:=\Delta M_{\text {conv }} / \dot{M}$.

Ideally, the properties of the helium convection zone could be studied through multi-dimensional hydrodynamic calculations. However, the large thermal diffusivity poses a formidable computational challenge since it makes the problem numerically stiff: the diffusive timescale is much shorter than the dynamical one, which leads to very short time steps if an explicit solver is used (unfortunately, most codes used for compressible convection are explicit). Any simulation would have only limited value unless it includes a sufficiently realistic treatment of the coupling between plasma and radiation.

In the presence of strong wind mass-loss, another consideration related to the He convective zone becomes important, due to the fact that it comprises only a tiny amount of mass. Convection can set in only if the turnover time $\tau_{\text {turn }} \simeq H_{\mathrm{P}} / v_{\mathrm{c}}$ is shorter than the time scale for which convection is predicted to prevail at a fixed Lagrangian mass shell inside the convection zone, $\tau_{\text {conv }}$, which is $\tau_{\text {conv }} \simeq \Delta M_{\text {conv }} / \dot{M}$. We find a critical mass-loss rate $\dot{M} \sim 10^{-6} M_{\odot} \mathrm{yr}^{-1}$, above which convection has no time to develop in the helium region, since the wind is removing an amount of mass equivalent to the mass of the convection zone before a convective eddy can turn over (see Table 1). For a metallicity $Z=0.02$, stars above $40 M_{\odot}$ cannot develop the He convection zone, and in a $20 M_{\odot}$ such a layer is convective only for 10-100 turnovers before convection moves to a lower mass coordinate. None of these concerns is significant for the iron convection zone (FeCZ hereafter), where convection is always fully developed. Moreover the convective velocities for the FeCZ are always found to be much higher than those in the helium convection zones. We disregard the occurrence of the helium convection zones unless it is explicitly mentioned.

\section{Results}

We calculated a grid of non-rotating stellar evolution sequences for initial masses between $5 M_{\odot}$ and $100 M_{\odot}$, at metallicities of $Z=0.02, Z=0.008$ and $Z=0.004$, roughly corresponding to the Galaxy, the LMC and the SMC, respectively. Additionally, we computed several models at lower metallicity. Since rapid rotation can change the properties of sub-surface convection (Maeder et al. 2008), we calculated a few rotating models to evaluate the effects of rotation on our results. These effects are discussed in Sect. 3.1.

Figures 2 and 3 show the evolution of the radial extent and location of the sub-surface convection zones in $20 M_{\odot}$ and $60 M_{\odot}$ models during the main sequence phase.

As outlined above, the He opacity bump at around $\log T \simeq 4.7$ is responsible for a convective zone which occurs close to the stellar surface and is very inefficient: only a very small fraction of the heat flux is transported by bulk motions in this region. The upper boundary is typically found at an optical depth in the range $2 \leq \tau \leq 10$, where $\tau$ is the Rosseland mean
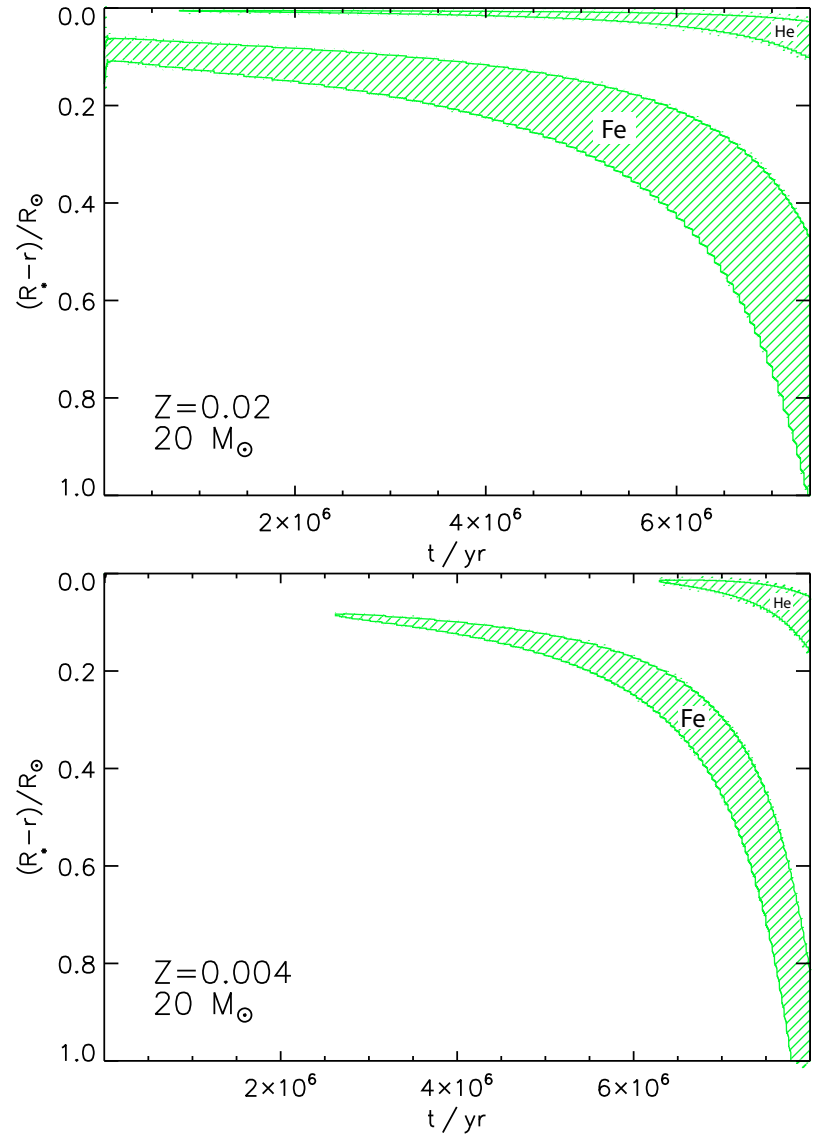

Fig. 2. Evolution of the radial extent of the helium and iron convective regions (hatched) as function of time, from the zero age main sequence to roughly the end of core hydrogen burning, for a $20 M_{\odot}$ star. The top of the plot represents the stellar surface. Only the upper $1 R_{\odot}$ of the star is shown in the plot, while the stellar radius itself increases during the evolution. Upper panel: the star has a metallicity of $Z=0.02$, and its effective temperature decreases from $35000 \mathrm{~K}$ to $25000 \mathrm{~K}$ during the main sequence phase. Lower panel: the star has a metallicity of $Z=0.004$, and its effective temperature decreases from $37000 \mathrm{~K}$ to $27000 \mathrm{~K}$ during the main sequence phase. The extent of the convection zones is smaller than in the case shown above, and the iron zone is absent for the first 2.5 million years.

optical depth. Below this convective zone, the Fe opacity bump at around $\log T \simeq 5.3$ is associated with a more efficient and extended convective region.

The radial extent of the $\mathrm{FeCZ}$ is quite substantial, i.e. a significant fraction of one solar radius, which corresponds typically to 2-10 pressure scale heights, comprising a mass on the order of $10^{-6} M_{\odot}$ to $10^{-5} M_{\odot}$, while the amount of mass between the top of the $\mathrm{FeCZ}$ and the stellar surface is around several times 


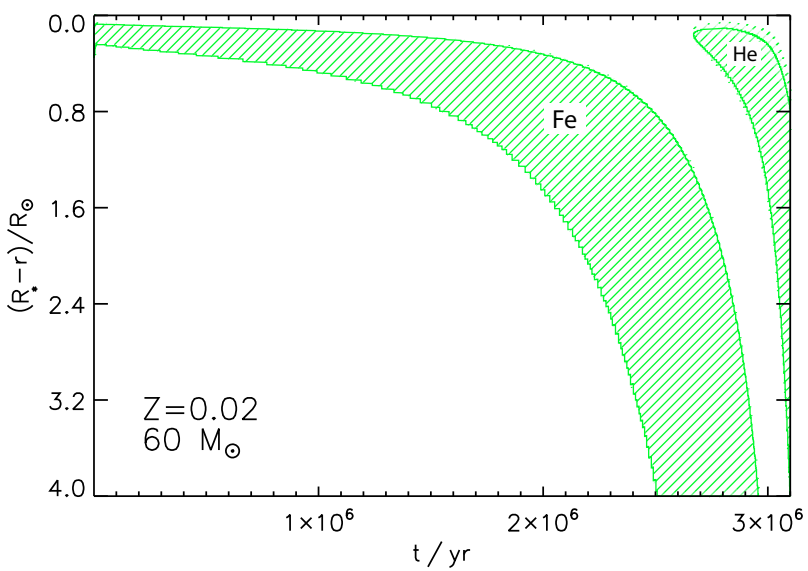

Fig. 3. Same as Fig. 2, but for a $60 M_{\odot}$ star at $Z=0.02$. Note the different vertical scale, spanning the upper $4 R_{\odot}$ of the star. The effective temperature decreases from $48000 \mathrm{~K}$ to $18000 \mathrm{~K}$ during the main sequence phase.

$10^{-7} M_{\odot}$ (cf. Table 1$)$. In the $20 M_{\odot}$ model the upper border of the FeCZ is located at $\tau \approx 140$ on the ZAMS, and at $\tau \approx 370$ on the cool side of the main sequence band. In the $60 M_{\odot}$ model the upper border at ZAMS is located at $\tau \approx 15$, reaching $\tau \approx 260$ during the late main sequence evolution. Convective velocities predicted by the MLT are on the order of $10 \mathrm{~s}$ of $\mathrm{km} \mathrm{s}^{-1}$, where more extended zones achieve higher velocities. For a quantitative analysis, we define an average convective velocity

$\left\langle v_{\mathrm{c}}\right\rangle:=\frac{1}{\alpha H_{\mathrm{P}}} \int_{R_{\mathrm{c}}-\alpha H_{\mathrm{P}}}^{R_{\mathrm{c}}} v_{\mathrm{c}}(r) \mathrm{d} r$

where $R_{\mathrm{c}}$ is the upper boundary of the convective zone, and where we set $\alpha=1.5$.

From Figs. 2 and 3, three trends for the extent of the subsurface convection zones are noticeable. First, with increasing time during the main sequence evolution, these zones become more extended, and are located deeper inside the stellar envelope. This is because the stellar envelope expands, and becomes cooler, while the temperature of the opacity peak remains nearly constant. In our $20 M_{\odot}$ model at $Z=0.02$, the mass of the $\mathrm{He}$ convective zone increases from about $10^{-9} M_{\odot}$ to $2 \times 10^{-7} M_{\odot}$, and that of the FeCZ is growing from $2 \times 10^{-6} M_{\odot}$ to $10^{-4} M_{\odot}$. For sufficiently hot models, the helium convection zones can even vanish (Fig. 2, lower panel). Second, comparing the $20 M_{\odot}$ and the $60 M_{\odot}$ model at $Z=0.02$ demonstrates that the FeCZ becomes more prominent for higher luminosity. This is because the opacity is not substantially changing among main sequence models at the same metallicity, such that a higher luminosity renders a larger portion of the envelope convectively unstable (both in radius and mass fraction). Our models show that the FeCZ disappears below a threshold luminosity of about $10^{4} L_{\odot}$ on the ZAMS at solar metallicity. Third, comparing the two $20 M_{\odot}$ models in Fig. 2 shows that the extent of the FeCZ, and its presence, depends on the metallicity. We find that for $Z=0.001$, it is completely absent below $40 M_{\odot}$, and at $Z=0.00001$ it does not occur for $M \leq 60 M_{\odot}$. In summary, our models predict an increase of the importance of the FeCZ for cooler surface temperature or lower surface gravity, for higher luminosity, and for higher metallicity.

While in the discussed range of luminosity and effective temperature, the average convective velocity $\left\langle v_{\mathrm{c}}\right\rangle$ is on the order of 1 to $10 \mathrm{~km} \mathrm{~s}^{-1}$ for the $\mathrm{FeCZ}$, we found that the average convective velocity $\left\langle v_{\mathrm{c}}\right\rangle$ in the He convective zone is always very low $\left(\lessgtr 1 \mathrm{~km} \mathrm{~s}^{-1}\right)$. Convection due to hydrogen recombination is absent; this dominates at lower effective temperatures than the ones studied here.

For our grid of stellar evolution models, we map the average convective velocity of the FeCZ (Eq. (6)) in the HR diagram for the three different metallicities (see Fig. 9, and Sect. 4.1.2). This figure displays the three qualitative trends of the iron zone we have just described.

- For given luminosity and metallicity, the average convective velocity near the upper boundary of the FeCZs increases with decreasing surface temperature. The convection zones are located deeper inside the star (in radius, not in mass), and the resulting larger pressure scale height leads to higher velocities. At solar metallicity and $10^{5} L_{\odot}$ (i.e. roughly at $\left.20 M_{\odot}\right)$ the velocities increase from just a few $\mathrm{km} \mathrm{s}^{-1}$ at the ZAMS to more than $10 \mathrm{~km} \mathrm{~s}^{-1}$ in the supergiant regime, where $\left\langle v_{\mathrm{c}}\right\rangle=2.5 \mathrm{~km} \mathrm{~s}^{-1}$ is achieved at $T_{\text {eff }} \simeq 30000 \mathrm{~K}$. At the lowest considered metallicity, the FeCZ is absent at the ZAMS at $10^{5} L_{\odot}$, and a level of $\left\langle v_{\mathrm{c}}\right\rangle=2.5 \mathrm{~km} \mathrm{~s}^{-1}$ is only reached at $T_{\text {eff }} \simeq 20000 \mathrm{~K}$.

- For fixed effective temperature and metallicity, the iron zone convective velocity increases with increasing luminosity, since a larger flux demanded to be convectively transported requires faster convective motions. Figure 9 in Sect. 4.1.2 also shows that there are threshold luminosities below which FeCZs do not occur, i.e., below about $10^{3.2} L_{\odot}, 10^{3.9} L_{\odot}$, and $10^{4.2} L_{\odot}$ for the Galaxy, LMC and SMC, respectively.

- The FeCZs become weaker for lower metallicities, since due to the lower opacity, more of the flux can be transported by radiation. The threshold luminosity for the occurrence of the FeCZ quoted above for $Z=0.02$ is ten times lower than that for $Z=0.004$. And above the threshold, for a given point in the HR diagram, the convective velocities are always higher for higher metallicity.

\subsection{Rotating models}

We considered two $20 M_{\odot}$ models with metallicity $Z=0.02$, one rotating at birth with an equatorial velocity of $250 \mathrm{~km} \mathrm{~s}^{-1}$ (corresponding to about $35 \%$ of the critical velocity) and one with $350 \mathrm{~km} \mathrm{~s}^{-1}$ (about $50 \%$ of the critical velocity). The evolution of the radial extent of sub-surface convection in the rotating models is very similar to the non-rotating case shown in Fig. 2. Also the convective velocities inside the FeCZ change only a few percent between rotating and non-rotating models, even if the rotating models show slightly higher convective velocity peaks (see Fig. 4). We conclude that rotation is not significantly affecting the structure and the properties of sub-surface convection in the vast majority of $\mathrm{OB}$ stars.

As pointed out by Maeder et al. (2008), the effects of rotation on sub-surface convection become substantial for stars rotating close to critical velocity. While stars rotating with such high velocities exist (e.g. Be stars), their number is modest. The study of sub-surface convection in these very fast rotators is interesting, but may require 2-dimensional stellar models, which is beyond the scope of this paper.

\section{Comparison with observations}

In the following, we investigate the idea that these sub-surface convection zones might be related to observable phenomena at the stellar surface. In particular, we investigate potential connections with microturbulence in massive stars, and discuss whether 


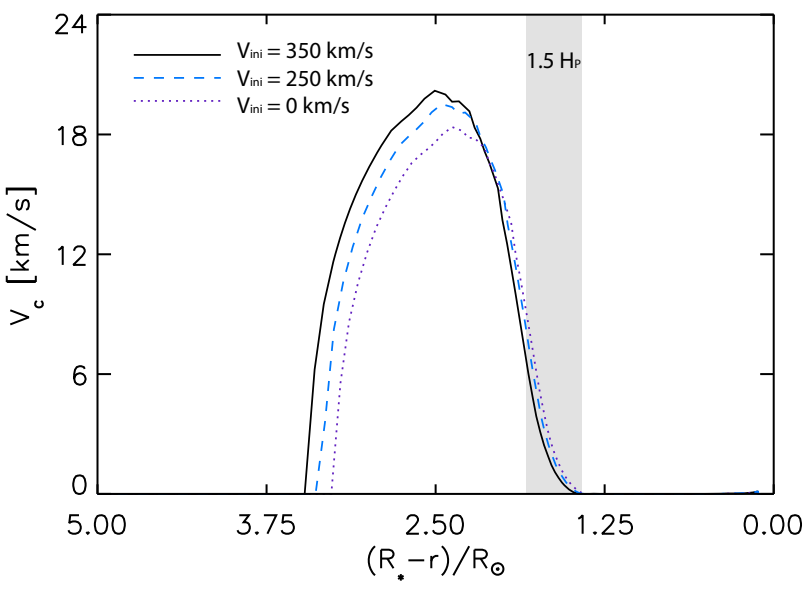

Fig. 4. Convective velocity in the $\mathrm{FeCZ}$ as function of radial distance from the stellar surface. The dotted line corresponds to a non-rotating $20 M_{\odot}$ model at $Z=0.02$, while the dashed and solid lines refer to the same model rotating at birth with $250 \mathrm{~km} \mathrm{~s}^{-1}$ and $350 \mathrm{~km} \mathrm{~s}^{-1}$ respectively. The values correspond to models having the same effective temperature $(\log T=4.339)$ and very similar luminosity $\left(\log L / L_{\odot}=5.04\right.$ for the non-rotating model and $\log L / L_{\odot}=5.03$ for the rotating ones). The gray band shows the upper 1.5 pressure scale heights of the $\mathrm{FeCZ}$, which is the region considered for the computation of $\left\langle v_{c}\right\rangle$, cf. Eq. (6). Convective velocities in the He convection zone are much lower than $1 \mathrm{~km} \mathrm{~s}^{-1}$ and are not visible in this plot.

small scale or large scale clumping in massive star winds, magnetic fields, and non-radial pulsations could be related to subsurface convection. For each point, we first briefly discuss the theoretical motivation, and then the corresponding observational evidence.

\subsection{Microturbulence}

\subsubsection{Theoretical considerations}

The convective cells in the upper part of a convection zone excite acoustic and gravity waves that propagate outward. The generation of sound waves by turbulent motions was first discussed by Lighthill (1952) and extended to a stratified atmosphere by Stein (1967) and Goldreich \& Kumar (1990). In a stratified medium, gravity acts as a restoring force and allows the excitation of gravity waves. For both acoustic and gravity waves, the most important parameter determining the emitted kinetic energy flux is the velocity of the convective motions. This is why, in the following, we use the average convective velocity $\left\langle v_{\mathrm{c}}\right\rangle$ as the crucial parameter determining the efficiency of sub-surface convection.

Goldreich \& Kumar (1990) showed that convection excites acoustic and gravity waves, resulting in maximum emission for those waves with horizontal wave vector $k_{\mathrm{h}} \sim 1 / H_{\mathrm{P}, \mathrm{c}}$ and angular frequency $\omega \sim v_{\mathrm{c}} / H_{\mathrm{P}, \mathrm{c}}$, where now $v_{\mathrm{c}}$ and $H_{\mathrm{P}, \mathrm{c}}$ are evaluated at the top of the convective region. They calculated that the amount of convective kinetic energy flux going into acoustic and gravity waves is

$F_{\mathrm{a} c} \sim F_{\mathrm{c}} M_{\mathrm{c}}^{15 / 2}$,

and

$F_{\mathrm{g}} \sim F_{\mathrm{c}} M_{\mathrm{c}}$

respectively, where we take $F_{\mathrm{c}} \sim \rho_{\mathrm{c}}\left\langle v_{\mathrm{c}}\right\rangle^{3}$ and $M_{\mathrm{c}}$ is the Mach number in the upper part of the convective region. Since convection in our models is subsonic, gravity waves are expected

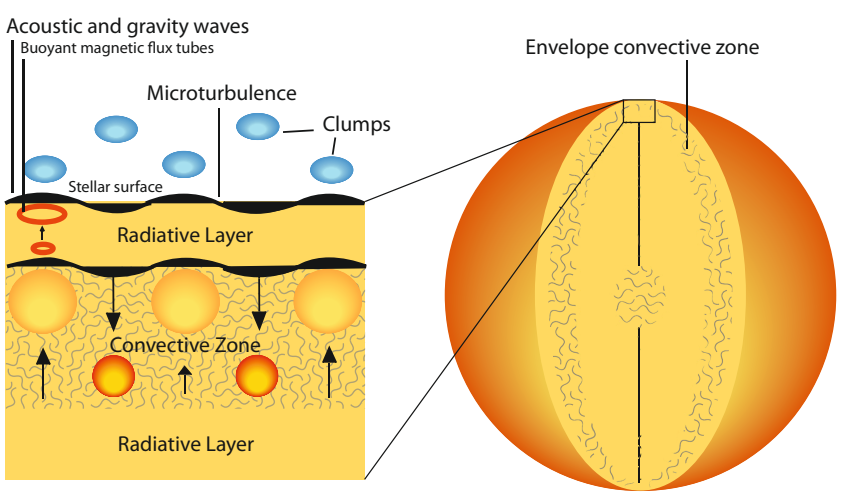

Fig. 5. Schematic representation of the physical processes connected to sub-surface convection. Acoustic and gravity waves emitted in the convective zone travel through the radiative layer and reach the surface, inducing density and velocity fluctuations. In this picture microturbulence and clumping at the base of the wind are a consequence of the presence of sub-surface convection. Buoyant magnetic flux tubes produced in the convection zone could rise to the stellar surface.

to extract more energy from the convective region than acoustic waves. These gravity waves can then propagate outward, reach the surface and induce observable density and velocity fluctuations (Fig. 5).

The Brunt-Vaisäla frequency in the radiative layer above the $\mathrm{FeCZ}$ is about $\mathrm{mHz}$. Molecular viscosity can only damp the highest frequencies, while wavelengths that will be resonant with the scale length of the line forming region should not be affected (see e.g. Lighthill 1967). This is the case for the gravity waves stochastically excited by convective motions: they can easily propagate through the sub-surface radiative layer, steepening and becoming dissipative only in the region of line formation.

Again, multi-dimensional hydrodynamic simulations would be the best way to compute the energy loss of these waves during their propagation through the radiatively stable envelope above the FeCZ, but this is beyond what we can presently do. We can, however, obtain an upper limit to the expected velocity amplitudes at the stellar surface, where we only consider the energy transport through gravity waves. The kinetic energy per unit volume associated with the surface velocity fluctuations $E_{\mathrm{s}}$ must be comparable to or lower than the kinetic energy density associated with the waves near the sub-surface convection zone, $E_{\mathrm{g}} \sim M_{\mathrm{c}} \rho_{\mathrm{c}}\left\langle v_{\mathrm{c}}\right\rangle^{2}$, or

$\frac{E_{\mathrm{g}}}{E_{\mathrm{s}}} \sim M_{\mathrm{c}}\left(\frac{\rho_{\mathrm{c}}}{\rho_{\mathrm{s}}}\right)\left(\frac{\left\langle v_{\mathrm{c}}\right\rangle}{v_{\mathrm{s}}}\right)^{2} \geq 1$,

where $\rho_{\mathrm{c}}$ is the density at the top of the convective region and $\rho_{\mathrm{s}}$ is the surface density, and $v_{\mathrm{S}}$ is the surface velocity amplitude. In this ratio we only consider energy density since the volume of the line forming region is comparable to the volume of the upper part of the convective zone. Therefore, we expect

$v_{\mathrm{s}} \leq\left\langle v_{\mathrm{c}}\right\rangle \sqrt{M_{\mathrm{c}} \frac{\rho_{\mathrm{c}}}{\rho_{\mathrm{s}}}}$.

In our models with well developed FeCZs, $\sqrt{M_{\mathrm{c}} \rho_{\mathrm{c}} / \rho_{\mathrm{s}}} \simeq 1$ (order of magnitude), and thus $v_{\mathrm{s}}$ and $\left\langle v_{\mathrm{c}}\right\rangle$ should be on the same order of magnitude. It is difficult to estimate the typical correlation length of the induced velocity field at the stellar surface, but a plausible assumption is that it is about one photospheric pressure scale height, $H_{\mathrm{P}, \mathrm{s}}$, given the proximity of the $\mathrm{FeCZ}$ to 
the surface and the fact that the horizontal wave vector of the emitted waves is $k_{\mathrm{h}} \sim 1 / H_{\mathrm{P}, \mathrm{c}}$.

\subsubsection{Observations}

The microturbulent velocity $\xi$ is defined as the microscale nonthermal component of the gas velocity in the region of spectral line formation:

$\Delta \lambda_{\mathrm{D}}=\frac{\lambda}{\mathrm{c}} \sqrt{\frac{2 \mathcal{R} T}{\mu}+\xi^{2}}$.

Assuming that the gas in this zone has a temperature only slightly different from the effective temperature, one finds empirically that the observed Doppler widths $\Delta \lambda_{\mathrm{D}}$ cannot be accounted for by the thermal motions alone (e.g. Cowley 1970). Regardless of which physical mechanism causes microturbulence, the process of spectral line fitting yields values of $\xi$ in hot massive stars between 0 and about $20 \mathrm{~km} \mathrm{~s}^{-1}$. In contrast, macroturbulence corresponds to velocity fluctuations which are coherent on a length scale larger than the radial extent of line forming regions. If indeed the length scale of the photospheric velocity fluctuations induced by the iron convection zone are on the order of the photospheric pressure scale height, then this length scale is also comparable to the radial extent of line forming regions, and it is difficult to decide whether the velocity fluctuations would be manifested as micro- or as macroturbulence, or both. Below, we compare our model predictions only to the case of microturbulence since this is the empirical parameter most extensively available in the literature.

Photospheric microturbulence is routinely required, e.g., to derive consistent surface abundances for one element from different photospheric absorption lines through stellar model atmospheres (among many others Rolleston et al. 1996; Hibbins et al. 1998; Vrancken et al. 2000). Unfortunately, differences in physical assumptions or atomic physics can require somewhat different microturbulent velocities for the same star in different studies. Here, we restrict our detailed comparison to the data of Trundle et al. (2007) and Hunter et al. (2008b) from the ESO VLT-FLAMES Survey of Massive Stars (Evans et al. 2005), since it comprises the largest available uniformly analyzed data set. In Fig. 6, we plot the microturbulent velocities derived for the LMC early B type stars analyzed by Hunter et al. (2008b) versus their projected rotational velocity. The error bar on the derived microturbulent velocities is usually quite big, $\pm 5 \mathrm{~km} \mathrm{~s}^{-1}$, and is often comparable to the measured quantity itself. There seems to be no positive correlation between $\xi$ and the apparent projected rotational velocity $v \sin i$. Though $v \sin i$ is plotted and not $v$ itself, the lack of a correlation in such a large data set (justifying the assumption of random orientation of the sample) argues against rotation as an important effect in triggering microturbulence in hot stars. To compare microturbulent velocities to properties of sub-photospheric convection we use only data obtained for slow rotators (i.e. $v \sin i<80 \mathrm{~km} \mathrm{~s}^{-1}$ ) as microturbulent velocities are more difficult to measure for faster rotators.

In Fig. 7, we show the microturbulent velocities for the LMC stars of Hunter et al. (2008b) versus the stellar surface gravity. Trends of the microturbulent velocity with $\log g$ have been previously reported for hot stars (e.g. Gies \& Lambert 1992; Hunter et al. 2007). The figure shows that indeed, for $\log g<3.2$, there is a clear trend. However, the luminosity coding in Fig. 7 suggests that this trend may be largely produced by the increase in convective velocity with increasing luminosity (Sect. 3). This figure displays a detection threshold of about

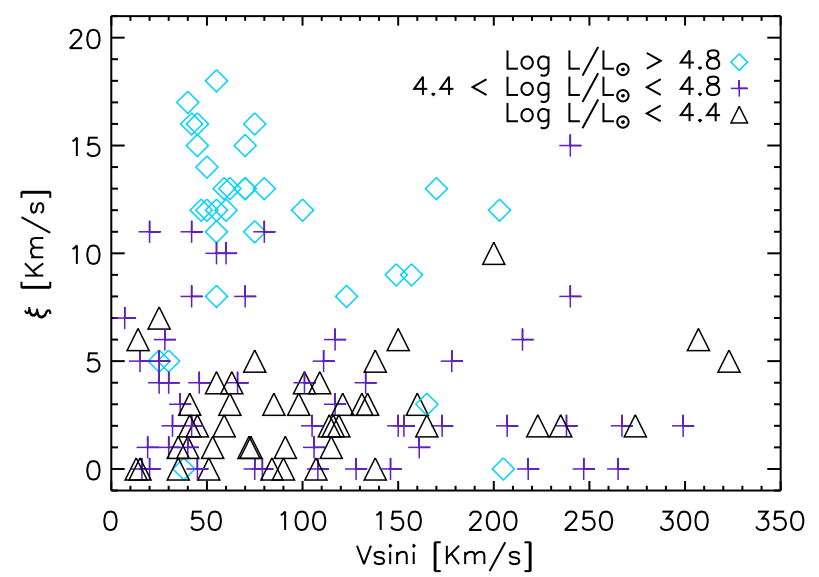

Fig. 6. Projected rotational velocity $v \sin i$ versus photospheric microturbulent velocity $\xi$ for the early B-type stars in the LMC analyzed by Hunter et al. (2008b). Different symbols refer to different luminosity intervals, as explained in the legend. The microturbulent velocities $\xi$ have typical uncertainties of about $\pm 5 \mathrm{~km} \mathrm{~s}^{-1}$. An uncertainty of $10 \%$ or $\pm 10 \mathrm{~km} \mathrm{~s}^{-1}$, whichever is the larger, should be considered for the rotational velocity measurements.

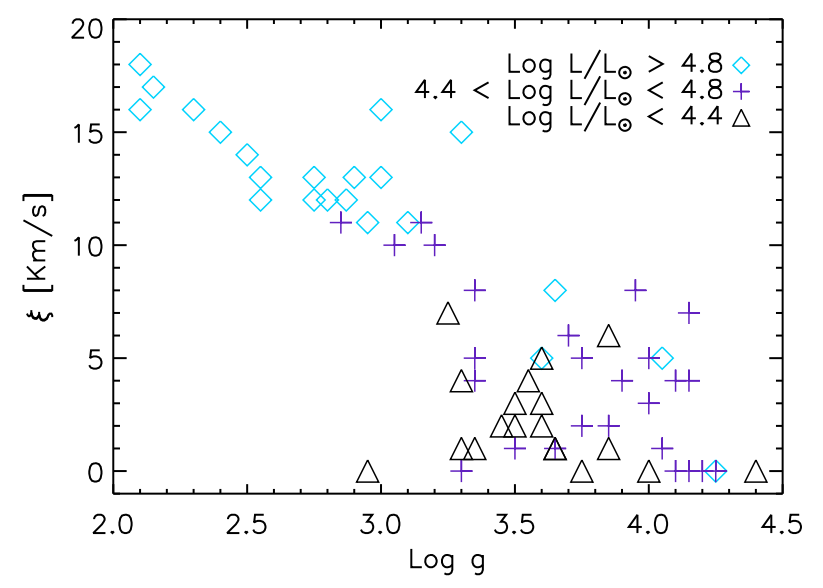

Fig. 7. Logarithm of surface gravity versus microturbulent velocity $\xi$ for the LMC early B-type stars studied by Hunter et al. (2008b); only stars with $v \sin i<80 \mathrm{~km} \mathrm{~s}^{-1}$ are considered here. Different symbols refer to different luminosity intervals, as explained in the legend. The microturbulent velocities $\xi$ have typical uncertainties of about $\pm 5 \mathrm{~km} \mathrm{~s}^{-1}$. For the surface gravity measurements an uncertainty of \pm 0.1 should be considered.

$10 \mathrm{~km} \mathrm{~s}^{-1}$ for the microturbulent velocities so in the following we restrict the comparison to $\xi \geq 10 \mathrm{~km} \mathrm{~s}^{-1}$.

In order to compare these observations to our model predictions, we evaluated the ratio of the kinetic energy in the form of gravity waves at the surface of the $\mathrm{FeCZ}$ to the kinetic energy of the surface velocity field, $E_{\mathrm{g}} / E_{\mathrm{s}}$ (Eq. (9)), assuming $v_{\mathrm{s}}=10 \mathrm{~km} \mathrm{~s}^{-1}$, in the HR diagram. Figure 8 shows two different iso-contours of this ratio; the stars of the LMC sample shown in Fig. 7 are over plotted. Notably, all but one of the LMC stars of Fig. 8 with $\xi>10 \mathrm{~km} \mathrm{~s}^{-1}$ are found in that part of the HR diagram where it is energetically possible that the FeCZ-induced gravity waves trigger a significant surface velocity field $\left(v_{\mathrm{s}}>10 \mathrm{~km} \mathrm{~s}^{-1}\right)$. Thus, a physical connection of the $\mathrm{FeCZ}$ with the observed microturbulent velocities appears energetically possible. Moreover, that the iso-contour line of 


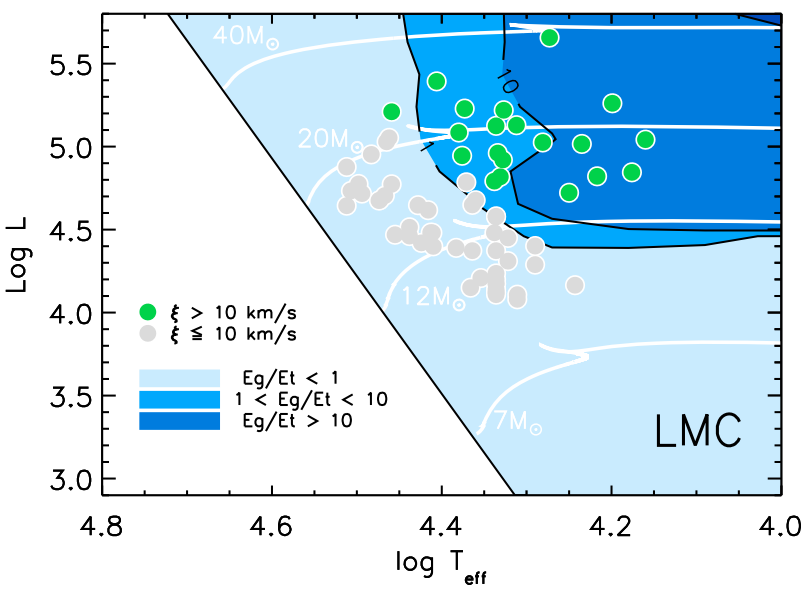

Fig. 8. Values of the ratio $E_{\mathrm{g}} / E_{\mathrm{s}}$ of the kinetic energy in the form of gravity waves above the iron convection zone, to the kinetic energy of the surface velocity field, as a function of the location in the HR diagram (see color scale). This plot is based on evolutionary models between $5 M_{\odot}$ and $100 M_{\odot}$ for LMC metallicity. We estimated the ratio $E_{\mathrm{g}} / E_{\mathrm{s}}$ as in Eq. (9), using a value $v_{\mathrm{s}}=10 \mathrm{~km} \mathrm{~s}^{-1}$ for the surface velocity amplitude. Over-plotted as filled circles are stars which have photospheric microturbulent velocities $\xi$ derived in a consistent way by Hunter et al. (2008b). Here, we use only data for stars with an apparent rotational velocity of $v \sin i<80 \mathrm{~km} \mathrm{~s}^{-1}$. The uncertainty in the determination of $\xi$ is typically $\pm 5 \mathrm{~km} \mathrm{~s}^{-1}$, which justifies our choice of $v_{\mathrm{s}}=10 \mathrm{~km} \mathrm{~s}^{-1}$. Solid white lines are reference evolutionary tracks. The full drawn black line corresponds to the zero age main sequence.

$E_{\mathrm{g}} / E_{\mathrm{s}}=1$ in Fig. 8 almost perfectly divides the observed sample in stars with significant $\left(\xi>10 \mathrm{~km} \mathrm{~s}^{-1}\right)$ and insignificant $\left(\xi<10 \mathrm{~km} \mathrm{~s}^{-1}\right)$ microturbulence is a further indication of such a physical connection.

Figure 9 shows the iso-contours in the HR diagram of the average convective velocity from our models in the upper layers of the iron convective zone, $\left\langle v_{\mathrm{c}}\right\rangle$ (cf., Sect. 4.1.1), at the three considered metallicities. We have over plotted the microturbulent velocities derived by Trundle et al. (2007) and Hunter et al. (2008b) as filled circles. Again, we distinguish between sample stars with significant $\left(\xi>10 \mathrm{~km} \mathrm{~s}^{-1}\right.$; Group A) and insignificant ( $\xi<10 \mathrm{~km} \mathrm{~s}^{-1}$; Group B) microturbulent velocities. Comparing the plot for the LMC in Fig. 9 with Fig. 8 identifies $\left\langle v_{\mathrm{c}}\right\rangle \simeq 2.5 \mathrm{~km} \mathrm{~s}^{-1}$ as a critical convection velocity to be able to trigger microturbulence. Interestingly, the contour of $\left\langle v_{\mathrm{c}}\right\rangle=2.5 \mathrm{~km} \mathrm{~s}^{-1}$ in our stellar models forms an almost perfect dividing line between Groups A and B for all three considered metallicities.

In fact, Fig. 9 provides evidence for all three trends found in the average convection velocity as function of stellar parameters (cf., Sect. 3) to be present also in the empirical data on microturbulent velocities. The LMC data shows that in the luminosity range $4.5<\log L / L_{\odot}<5.5$ microturbulence is found only for $T_{\text {eff }} \lesssim 25000 \mathrm{~K}$. The data for all three metallicities clearly suggests a key role of the luminosity, as the stars with $\xi>10 \mathrm{~km} \mathrm{~s}^{-1}$ are the most luminous ones in each sub sample. And finally, the stars with high microturbulent velocities are all comfortably above our theoretical contour line corresponding to $\left\langle v_{\mathrm{c}}\right\rangle=2.5 \mathrm{~km} \mathrm{~s}^{-1}$. As the latter trends toward the upper right corner of the HR diagram for lower metallicity, the metallicity dependence is also confirmed by the empirical data.

Lyubimkov et al. (2004) studied microturbulence in a sample of 100 Galactic early B stars. Interestingly, they found significant microturbulent velocities (i.e., clearly above $5 \mathrm{~km} \mathrm{~s}^{-1}$ )
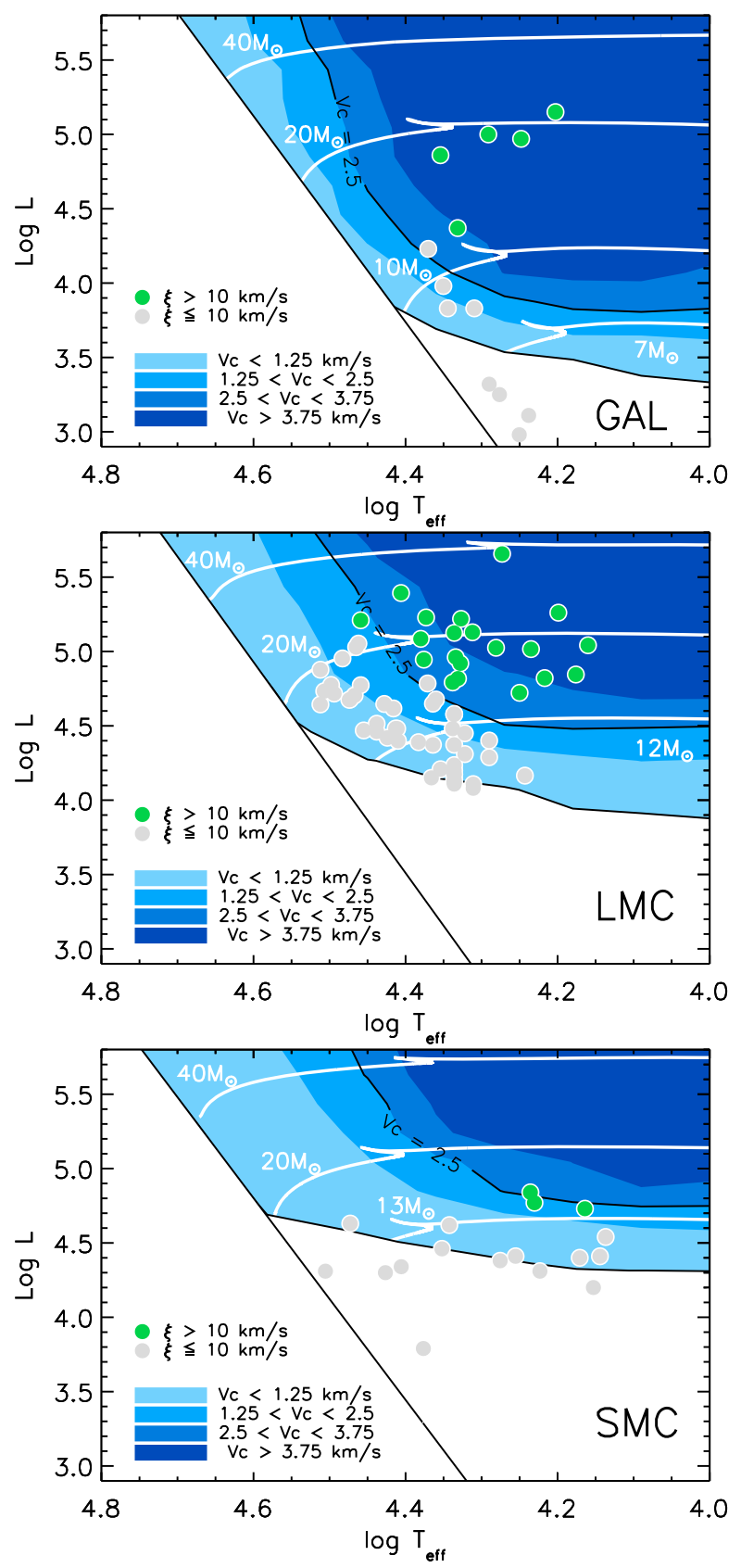

Fig. 9. Average convective velocity within 1.5 pressure scale heights of the upper border of the iron convection zone in our models, as function of the location in the HR diagram (see color scale), based on evolutionary models between $5 M_{\odot}$ and $100 M_{\odot}$ (white lines), for three metallicities corresponding to the Galaxy (top panel), the LMC (middle), and the SMC (bottom). The full drawn black line corresponds to the zero age main sequence. Over-plotted as filled circles are photospheric microturbulent velocities $\xi$ derived in a consistent way for hot massive stars by Trundle et al. (2007) and Hunter et al. (2008b). Here, we use only data for stars with an apparent rotational velocity of $v \sin i<80 \mathrm{~km} \mathrm{~s}^{-1}$. The uncertainty in the determination of $\xi$ is typically $\pm 5 \mathrm{~km} \mathrm{~s}^{-1}$.

in the mass range $7 \ldots 11 M_{\odot}$ for stars with a relative age on the main sequence of $t / \tau_{\mathrm{MS}}>0.8$, and in the range $12 \ldots 19 M_{\odot}$ for $t / \tau_{\mathrm{MS}}>0.25$, but only insignificant microturbulent velocities for younger or less massive stars. Again, these results appear to agree with Fig. 9 up to a remarkable quantitative level.

In summary, our comparison provides evidence for a physical connection of microturbulence in hot star photospheres with the existence and strength of a sub-photospheric FeCZ. 
If microturbulence has a physical origin and is not just a fudge factor, the pressure and energy terms associated with such a velocity field should be included in the calculations of atmospheric models of massive stars. Hubeny et al. (1991) have investigated part of these effects by accounting for a constant microturbulent velocity in the pressure term only. They find that for stars with conspicuous $\xi$ values (of $25 \mathrm{~km} \mathrm{~s}^{-1}$ ) the inclusion of the pressure term leads to higher values of the surface gravity, which can reduce the mass discrepancy for $\mathrm{O}$ stars and O-type central stars of planetary nebula. A similar approach was also studied by Smith \& Howarth (1998). The impact on gravity discussed by Hubeny et al. (1991) is likely an upper limit to the effect as, first, the $\xi$ values are in most cases less than $25 \mathrm{~km} \mathrm{~s}^{-1}$, and, second, a positive gradient in the atmospheric $\xi(r)$ would decrease the pressure gradient due to microturbulence but, to date, the radial stratification of the microturbulent velocity in the atmospheres of hot massive stars has not been studied in detail. From a theoretical perspective, investigating $\xi(r)$ requires hydrodynamic simulations of the stellar atmosphere, including the presence of sub-surface convection.

The mass discrepancy in massive stars is a well documented problem (see for example Herrero et al. 1992; Lennon et al. 2003; Trundle \& Lennon 2005; Massey et al. 2005; Mokiem et al. 2007). It is typically found that the difference between spectroscopic mass and evolutionary mass is most pronounced in supergiants. In main sequence stars it may not be present at all, but see Hunter et al. (2008b). Given that microturbulent velocities are highest in supergiants (see Fig. 7) an empirical correlation between mass discrepancy and microturbulent velocity is to be expected and is shown in Fig. 10 using data analysed by Trundle et al. (2007) and Hunter et al. (2008b). If indeed microturbulence is related to subsurface convection and supergiants have intrinsically higher microturbulent velocities than dwarfs (see Sect. 3) potentially part of the gradient in Fig. 10 may be explained by the effect discussed by Hubeny et al. (1991).

\subsection{Non-radial pulsation}

\subsubsection{Theoretical considerations}

In our discussion thus far we have considered only the propagation of running waves, it is possible that the stochastic convective motions can also excite standing waves, i.e. high-order nonradial pulsations. For example, stochastic excitation is thought to be the cause of the Solar oscillations (Ulrich 1970; Leibacher $\&$ Stein 1971). It may thus be possible that the FeCZ excites non-radial pulsations in hot early-type stars.

Several classes of OB star models are found to be linearly unstable against non-radial pulsations, among which are the $\beta$ Cephei stars and the slowly pulsating B stars (e.g., Dupret 2001; Pamyatnykh 1999). The key ingredient required for the pulsational instability is the iron opacity peak described in Sect. 2. As convection is not required to produce the pulsations in these models, it is not considered in detail as excitation mechanism (Dziembowski 2008). It is conceivable that the convective excitation could modify the predicted pulsation spectrum and/or extend the instability region of certain linear instabilities. The convective kinetic energy flow into waves could be predominantly directed into those modes for which instability is predicted in the models. In certain parts of the HR diagram, one may thus suspect an intricate connection between the occurrence of a sub-photospheric iron convection zone and the properties of non-radial pulsations.

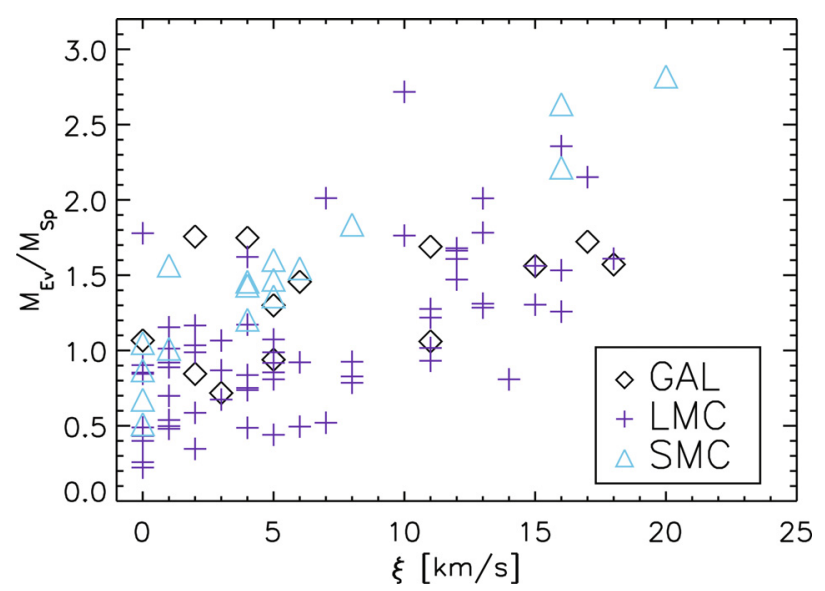

Fig. 10. Values of the mass discrepancy (evolutionary mass divided by spectroscopic mass) as function of microturbulent velocity in the sample of B stars analysed by Trundle et al. (2007) and Hunter et al. (2008b). Here, we use only data for stars with an apparent rotational velocity of $v \sin i<80 \mathrm{~km} \mathrm{~s}^{-1}$.

Non-radial pulsations have also been considered as the origin of various observed small scale (e.g., line profile variability, Fullerton et al. 1996, 1997) and large scale phenomena (e.g., so called discrete absorption components, Prinja \& Howarth 1988; Massa et al. 1995; Kaper et al. 1997; Prinja et al. 2002) at the surface or in the wind of massive OB stars. Non-radial g-mode pulsations were also recently proposed as the origin of observable macroturbulence in massive B type stars (Aerts et al. 2008). In Fig. 11 we compare the regions where strange mode, g-mode, and p-mode pulsations are predicted to occur in the HR diagram with the region where our models predict a strong FeCZ. Pulsations appear to be almost ubiquitous when all types of variables are accounted for. The strange mode pulsators are predicted to cover the HR diagram at high luminosity, where we plotted only the predictions for the radial strange modes of Kiriakidis et al. (1993); high-order non-radial strange modes seem to be omnipresent as well for stars above $40 M_{\odot}$ or so (Glatzel \& Mehren 1996). Non-radial g-mode pulsators are predicted by Saio et al. (2006) in the B supergiant region. And radial and low order non-radial $\mathrm{p}$-modes are predicted for the $\beta$ Cephei regime by Deng \& Xiong (2001) and by Pamyatnykh (1999) and Saio et al. (2006) for a considerably larger region in the HR diagram. At lower metallicity, many of the predicted areas in the HR diagram are smaller (cf., Kiriakidis et al. 1993; Deng \& Xiong 2001) but the general picture is still incomplete.

\subsubsection{Observations}

Observationally, the classical $\beta$ Cephei stars are concentrated in the region predicted by Deng \& Xiong (Stankov \& Handler 2005), while the B supergiant non-radial g-mode pulsators overlap with the prediction of Saio et al. (2006) but extend to an effective temperature of $\sim 10000 \mathrm{~K}$ (Lefever et al. 2007). Pulsations are also found for the most luminous stars (e.g., the $\alpha$ Cygni-variables; van Leeuwen et al. 1998), but there is now no clear evidence for strange mode pulsators. Comparing the prediction for the FeCZ with that for pulsational instability (Fig. 11) shows two things. Firstly, the FeCZ-region is much larger than any region for a particular pulsational instability. Thus, distinguishing whether a certain observational feature is caused by a particular pulsational instability by the $\mathrm{FeCZ}$ might, in principle, be possible, since the area in the HR diagram where the latter occurs but the pulsational instability does not is relatively large. 


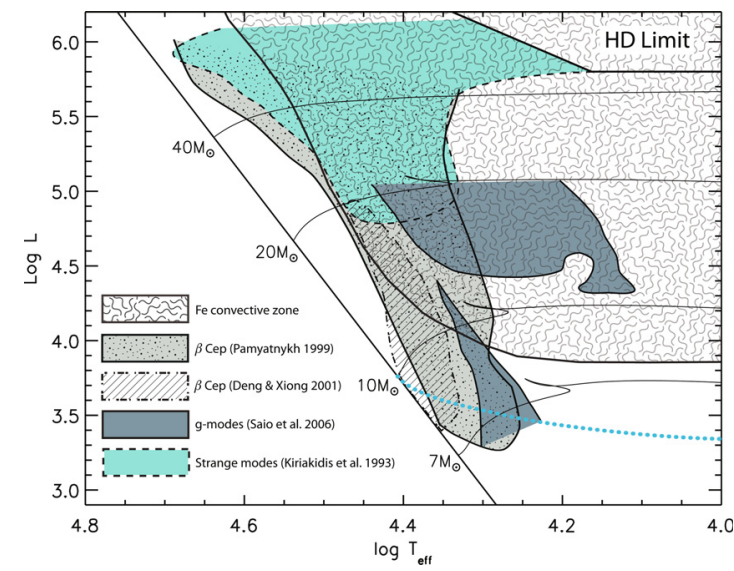

Fig. 11. The plot shows regions of the HR diagram where pulsational instabilities are predicted, compared to our calculations for the occurrence of iron convection. The cloudy region marks the presence of iron sub-surface convection with $\left\langle v_{\mathrm{c}}\right\rangle \geq 2.5 \mathrm{~km} \mathrm{~s}^{-1}$, while the dotted, blue line divides regions of the HR diagram where iron convection is present (above) from regions where it is absent (below). Different modes of instabilities are shown with different colors and different contour line styles, as explained in the legend. Evolutionary tracks between $7 M_{\odot}$ and $40 M_{\odot}$ are plotted as a reference. The straight, full drawn black line corresponds to the zero age main sequence. The Humphrey-Davidson limit is also plotted for reference (top-right corner).

Secondly, some regions exist where (so far) no pulsations are predicted but the FeCZ in our models is strong, or where, vice versa, pulsations are predicted but the $\mathrm{FeCZ}$ is weak or absent.

Comparing Figs. 11 with 9, where we show the observations of microturbulence and the $\mathrm{FeCZ}$ predictions, it is unlikely that microturbulence is associated with a particular pulsational instability. Strong microturbulence is observed at too low a luminosity to be attributable to strange mode pulsations alone, while p-mode pulsators are found where microturbulence seems not to occur. Concerning the g-mode pulsators the situation is less clear. Figure 11 shows that, at solar metallicity, gmode pulsations for post-main sequence stars are expected only in a rather narrow luminosity interval. Unfortunately, the five Galactic stars shown in Fig. 5 for which strong microturbulence is derived are all inside this luminosity range, so they cannot distinguish between a pulsational or $\mathrm{FeCZ}$ origin of microturbulence. However, looking at the LMC data, stars above the gmode luminosity upper limit with microturbulence are found; whether or not corresponding stellar models are g-mode unstable is currently not known. A connection of microturbulence with non-radial pulsations is thus not impossible, but it is also not very likely.

Comparing Fig. 11 with the discrete absorption components (DACs) found in 200 Galactic O stars above $\sim 20 M_{\odot}$ by Howarth \& Prinja (1989) all the way to the zero age main sequence, seems to argue against non-radial pulsations as the origin of the DACs phenomenon (see also Sect. 4.4).

\subsection{Wind clumping}

\subsubsection{Theoretical considerations}

Observational evidence exists for stellar wind inhomogeneities on small and on large scales. While the latter will be discussed in Sect. 4.4, here we consider only small scale wind structure, or wind clumping. In Sect. 4.1, we discussed that waves produced by the FeCZ could lead to velocity fluctuations at the stellar surface. In order to induce wind clumping, those waves should induce density fluctuations at the stellar surface. Through the occurrence of porosity or shifts in the ionisation balance of the gas the mass-loss rate may be affected. For this to happen, the amplitude of the velocity fluctuations at the surface should be on the same order of the sound speed. Alternatively, the velocity fluctuations might directly affect the local mass-loss rate through the Doppler effect, if the amplitude of the velocity fluctuations is on the same order as the speed of the wind flow, which, at the base of the wind, is approximately the sound speed. As the sound speed at the surface in our massive main sequence models is on the order of a few times $10 \mathrm{~km} \mathrm{~s}^{-1}$, we consider here those stellar models potentially capable to produce wind clumping for which the convective velocities in the upper part of the FeCZ $\left\langle v_{\mathrm{c}}\right\rangle \geq 2.5 \mathrm{~km} \mathrm{~s}^{-1}$, as this allows energetically to have surface velocity amplitudes above $\sim 10 \mathrm{~km} \mathrm{~s}^{-1}$ (cf. Sect. 4.1).

Assuming the horizontal extent of the clumps to be comparable to the sub-photospheric pressure scale height $H_{\mathrm{p}}$, we may estimate the number of convective cells by dividing the stellar surface area by the surface area of a convective cell finding that it scales with $\left(R / H_{\mathrm{P}}\right)^{2}$. For our main sequence $\mathrm{O}$ star models in the mass range $20-60 M_{\odot}$, we find pressure scale heights in the range $0.04-0.24 R_{\odot}$, corresponding to a total number of clumps in the range $6 \times 10^{3}-6 \times 10^{4}$. In principle, this might be testable through linear polarization variability measurements, which can probe wind asphericity at the very base of the wind (Davies et al. 2007).

\subsubsection{Observations}

Evidence has been accumulating that the winds of massive stars may be subject to small scale clumping. So far this is best documented for Wolf-Rayet (WR) stars, where line variability on time scales of minutes to hours is thought to constitute direct evidence of outflows that are clumped already in the acceleration zone near the base of the wind (Lépine \& Moffat 1999). This clumping may be part of the explanation for the wealth of intricate detail seen in nebulae around WR stars (Grosdidier et al. 1998). Recently, Lepine \& Moffat (2008) reported spectroscopic variability in the Of supergiants $\zeta$ Pup (see also Eversberg et al. 1998) and HD 93 129A. The amplitude of the variation (at the $1-3 \%$ level) is similar as in WR stars supporting the notion that clumping is not restricted to WR stars.

Indeed, evidence that $\mathrm{O}$ star winds are clumped is given by, among others, Puls et al. (2006). These authors investigate the clumping behavior of the inner wind (inside about two stellar radii) relative to the clumping in the outer wind (beyond tens of stellar radii) of a large sample of supergiant and giant stars. They find that in stars that have strong winds, the inner wind is more strongly clumped than the outer wind, whereas those having weak winds have similar clumping properties in the inner and outer regions. Their analysis only allows for such a relative statement. In principle, for weak winds the outer part could be homogeneous. If so, weak winds are not clumped. In any case, strong winds - identified as such if $\mathrm{H} \alpha$ is seen in emission are clumped near the base of the wind. A measure of the degree of clumping is the clumping factor $f_{\mathrm{cl}}=\left\langle\rho^{2}\right\rangle /\langle\rho\rangle^{2} \geq 1$ where angle brackets denote (temporal) average values (e.g. Puls et al. 2006).

Apparently, this type of radial behavior is not consistent with hydrodynamical predictions of the intrinsic, self-excited linedriven instability (Runacres \& Owocki 2002, 2005). Such models predict a lower clumping in the inner wind than the outer wind. Moreover, if there was any dependence on wind density predicted at all, optically thin winds should be more strongly 
clumped than optically thick winds (Owocki \& Puls 1999; Puls et al. 2006). Therefore, the findings on the radial clumping behavior in $\mathrm{O}$ stars may point to an additional excitation mechanism of wind structure.

Figure 12 shows that the $\mathrm{O}$ stars investigated by Puls et al. (2006) populate the regime in the HR diagram in which our models predict the average convective velocity in the top part of the FeCZ to change from a few to over $2.5 \mathrm{~km} \mathrm{~s}^{-1}$, indicating that surface velocity fluctuations on the order of the local sound speed are possible (cf. Sect. 4.3.1). Though the part of the HR diagram that is covered by the sample is limited $\left(4.46 \lesssim \log T_{\text {eff }} \lesssim 4.66 ; 5.29 \lesssim \log L / L_{\odot} \lesssim 6.26\right)$, the trend is such that stars with relatively strong clumping in the inner winds are in a regime where $\left\langle v_{\mathrm{c}}\right\rangle$ is higher. A correlation between clumping at the base of the wind and $\left\langle v_{\mathrm{c}}\right\rangle$, i.e., between wind clumping and the properties of the $\mathrm{FeCZ}$, appears therefore possible. To further test the idea that the $\mathrm{FeCZ}$ produces wind clumping at the wind base for sufficiently luminous and cool stars it would be desirable to derive the radial clumping profiles for cooler (i.e. B-type) stars. If correct, such stars, both the ones with weak and strong winds, should have relatively strong clumping at the base of the wind.

To derive the spatial scale of the wind clumps from linear polarimetry has not yet been possible for main sequence OB stars. A limitation is that this technique requires very high signalto-noise observations (see discussion in Harries et al. 2002). Luminous Blue Variables (LBVs) however provide a more appropriate category of objects to test wind clump sizes, because of a combination of higher mass-loss rates, and lower wind velocities than for O stars (Davies et al. 2005). Indeed, Davies et al. (2007) show that in order to produce the observed polarization variability of P Cygni, the wind should consist of about $\sim 1000$ clumps per wind flow-time $\left(\tau_{\mathrm{fl}} \equiv R_{\star} / v_{\infty}\right)$. To see whether this observational result is compatible with sub-surface convection causing wind clumping, we considered the sub-surface convective regions of a massive star model with global properties similar to those of P Cygni (initial mass $60 M_{\odot}, \log L / L_{\odot}=5.9$, and $\log T=18000 \mathrm{~K}$ ). As a result of the lower gravity, the pressure scale height in the FeCZ in this model is about $4 R_{\odot}$, which is much bigger than in our $\mathrm{O}$ star models. Consequently, the same estimate for the number of clumps as done for the main sequence models in Sect. 4.3.1 yields about 500 clumps per wind flow time, a number which is quite comparable to that derived for P Cygni observationally (about $10^{3}$ clumps per wind flow time).

Finally, Fullerton et al. (1996) have conducted a spectroscopic survey of $\mathrm{O}$ stars and observed intrinsic absorption line profile variability (LPVs) for about $77 \%$ of their sample. They report an increase of incidence and amplitude of variability with increasing stellar radius and luminosity, as well as no statistically significant line profile variability for dwarfs earlier than $\mathrm{O}$. While Fullerton et al. attempt to relate their findings to the predictions of strange-mode pulsation in $\mathrm{O}$ stars by Kiriakidis et al. (1993), a comparison of their results (see their Fig. 13) with the occurrence of sub-surface convection as depicted in Fig. 9 indicates the possibility of a physical connection between line profile variability and sub-surface convection in $\mathrm{O}$ stars.

\subsection{Magnetic fields}

\subsubsection{Fields from iron convection zones}

In solar-type stars, surface convection zones modified by the stellar rotation are thought of being capable of producing a magnetic field through the so called $\alpha \Omega$-dynamo (Parker 1975;
Spiegel \& Weiss 1980; Spiegel \& Zahn 1992). The FeCZ in our massive main sequence stellar models has a spatial extent similar to the Solar convection zone, although its mass is much smaller, and OB stars are rapid rotators, so it is possible that a dynamo may also work in the envelopes of OB stars. If so, the magnetic field may be able to penetrate the radiatively stable layer above the $\mathrm{FeCZ}$, and dynamically significant field strengths might be achievable. To this end, we follow the model by MacGregor \& Cassinelli (2003) for the rise of buoyant magnetic flux tubes generated at the edge of the convective core of massive stars through their radiative envelope and apply this model to the $\mathrm{FeCZ}$ and the overlying radiative layer. The magnetic field strength $B_{0}$ in the iron convection zone is estimated assuming equipartition of kinetic energy density and magnetic energy density inside the convective layers:

$B_{0} \simeq 2 v_{\mathrm{c}} \sqrt{\pi \rho}$

which, for our $60 M_{\odot}$ star at $Z=0.02$, reaches about $B_{0} \simeq$ $2700 \mathrm{G}$ inside the iron convective zone. The surface field $B_{\mathrm{S}}$ is then obtained by multiplying this number with the ratio of the surface density $\rho_{\mathrm{s}}$ and the density in the FeCZ $\rho_{0}$, i.e. $B_{\mathrm{s}} \simeq$ $B_{0} \rho_{\mathrm{s}} / \rho_{0} \simeq 60 \mathrm{G}$. Similarly, for the $20 M_{\odot}$ model at $Z=0.02$ we obtain $B_{0} \simeq 1400 \mathrm{G}$ and $B_{\mathrm{s}} \simeq 10 \mathrm{G}$. Although at the surface, the magnetic pressure in the flux tubes is only on the order of a few percent of the total pressure, it is on the same order as the gas pressure and could thus lead to considerable horizontal density differences. Compared to the situation envisioned by MacGregor \& Cassinelli (2003), who found that the rise time of the flux tubes from the edge of the convective core to the stellar surface can be comparable to the main sequence life time (but see also MacDonald \& Mullan 2004), the rise time of the flux tubes from the FeCZ to the surface is much shorter. And while the initial magnetic field strength at the edge of the convective core can be considerably higher than our values of $B_{0}$, the surface fields obtainable from the sub-surface convection zones are higher, due to the much lower density contrast between convection zone and surface in this case.

As a consequence, even though we are far from a detailed picture, it seems conceivable that the FeCZs in massive main sequence stars produce localized magnetic fields at their surface. The interaction of the stellar wind with the localized surface magnetic fields could enhance the rate at which the wind induces a loss of stellar angular momentum. Furthermore, co-rotating density patterns in the outflowing wind could be produced by these local magnetic spots.

Rotation may play an important role in the dynamo process, possibly resulting in the appearance of stronger fields at the surface for faster rotating stars. To estimate this effect, a dynamo model accounting for the differential rotation needs to be implemented in the stellar evolution calculations. This will be discussed in a subsequent paper.

\subsubsection{Observations}

Surface magnetic fields have been linked to several observed phenomena in $\mathrm{OB}$ stars, e.g. discrete absorption components (DACs) in UV resonance lines (e.g., Prinja \& Howarth 1988; Massa et al. 1995; Kaper et al. 1997; Prinja et al. 2002), which are thought to diagnose large scale coherent wind anisotropies (Cranmer \& Owocki 1996; Lobel \& Blomme 2008), or the less coherent line profile variability mentioned above (Fullerton et al. 1996, 1997). Also non-thermal X-ray emission of OB main sequence stars has been proposed to relate to surface magnetic 


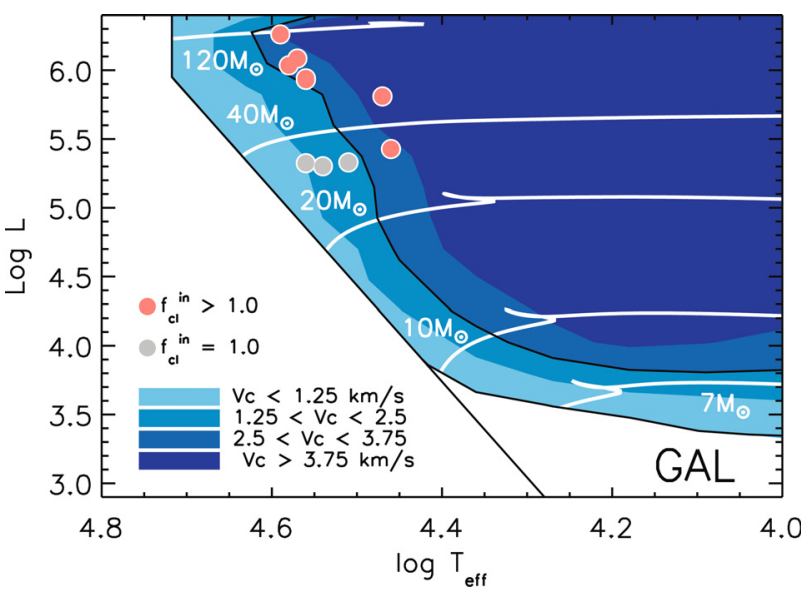

Fig. 12. Average convective velocity within 1.5 pressure scale heights of the upper border of the iron convection zone in our models, as function of the location in the HR diagram (see color scale), based on evolutionary models between $5 M_{\odot}$ and $120 M_{\odot}$ (white lines) at solar metallicity. The full drawn black line corresponds to the zero age main sequence. Over-plotted as filled circles are observations of the clumping factor $f_{\mathrm{cl}}^{\text {in }}$ (see text for definition) in the winds of $\mathrm{O}$ stars, according to Puls et al. (2006). The data shown here corresponds to objects with wellconstrained clumping parameters. Note the different luminosity range with respect to Fig. 9.

fields (e.g., Babel \& Montmerle 1997; ud-Doula \& Owocki 2002).

A connection of the FeCZ in massive stars with the phenomena mentioned above has not yet been considered. However, such a connection becomes testable through our results. While in our comparison to observed microturbulence presented above we discussed when sub-surface convection may lead to detectable surface velocity fluctuations, the presence of surface magnetic fields may simply depend on whether an FeCZ is present in the star or not. Looking at Fig. 9, we see that in our models the FeCZ is absent for luminosities below about $10^{3.2} L_{\odot}$, $10^{3.9} L_{\odot}$, and $10^{4.2} L_{\odot}$ for the Galaxy, LMC and SMC, respectively. If DACs or line profile variability were produced by magnetic flux tubes generated in the $\mathrm{FeCZ}$, those phenomena would not be expected for OB stars below those luminosities. Howarth \& Prinja (1989) find DACs in nearly all O stars (97\%) in a large Galactic sample, with $\log L / L_{\odot}>4.5$ and with effective temperatures as high as the zero-age main sequence values of stars above $\sim 20 M_{\odot}$. Since those stars are well above the luminosity threshold for the occurrence of the iron convection at Galactic metallicity, these observations do not exclude DACs being due to $\mathrm{FeCZ}$ induced B-fields. Also, all eleven early B supergiants with DACs in the sample of Prinja et al. (2002) are predicted to have strong FeCZ by our results. Notably, between about $20 M_{\odot}$ and $40 M_{\odot}$, stars close to the zero-age main sequence are not predicted to be pulsationally unstable (cf. Fig. 11), which may be in conflict with pulsations as the origin for DACs.

\subsubsection{Other types of fields}

It may be interesting to briefly compare the expectation from surface magnetic fields produced via the $\mathrm{FeCZ}$ to that for fields produced by other means. Surface fields produced by convective cores (Schuessler \& Paehler 1978; Charbonneau \& MacGregor 2001; MacGregor \& Cassinelli 2003) have been proposed to relate to the same phenomena as those mentioned above, even if for massive stars the buoyant rise of magnetic fields from the convective core seems to be unlikely (MacDonald \& Mullan 2004). In contrast to the sub-surface FeCZ, convective cores are prevalent in all stars above about $1.2 M_{\odot}$. It has been found that the longer lifetime of stars of lower mass may favor the drift of fields produced in the core to the surface (Schuessler \& Paehler 1978; MacGregor \& Cassinelli 2003). Therefore, the expected trend is opposite to that found for fields produced by the $\mathrm{FeCZ}$, where surface fields may occur only for stars above a critical mass (or luminosity), and stronger fields are found for more massive stars.

On the other hand, in contrast to fields from the FeCZ, magnetic flux tubes produced in the core may carry CNO-processed material to the surface. This might thus constitute a mechanism to explaining nitrogen enrichment in slowly rotating early B stars (Morel et al. 2006, 2008; Hunter et al. 2008a). Strong fossil magnetic fields are thought to persist in only a fraction of massive stars (Ferrario \& Wickramasinghe 2005; Braithwaite \& Spruit 2004), and may lead to, among other phenomena, highly anomalous surface chemical compositions, wind confinement, and variable X-ray emission (e.g., Wade et al. 2006; Townsend et al. 2005). Those strong features can clearly not be produced by fields originating from the FeCZs.

Finally, magnetic fields produced in differentially rotating massive stars by the Spruit-Taylor dynamo (Spruit 2002) may transport angular momentum and chemical species (cf., Heger et al. 2005). These fields are predominantly of toroidal geometry and would quickly decay near the stellar surface, and are thus not thought to lead to observable fields at the stellar surface (but see also Mullan \& MacDonald 2005).

\section{Concluding remarks}

Hot luminous stars show a variety of phenomena at their photosphere and in their winds which still lack a clear physical interpretation at this time. Among these phenomena are photospheric turbulence, spectral line variability (DACs and LPVs; see Sect. 4), wind clumping, variable or constant non-thermal $\mathrm{X}$-ray and radio emission, chemical composition anomalies, and intrinsic slow rotation. In the previous section, we argued that the iron convection zone could be responsible for various of these phenomena.

We proposed in Sect. 4.1 that a physical connection may exist between microturbulence in hot star atmospheres and a subsurface FeCZ. The strength of the FeCZ is predicted to increase with increasing metallicity $Z$, decreasing effective temperature $T$ and increasing luminosity $L$ (Sect. 3 ), and all three predicted trends are reflected in the observational data. This suggests that microturbulence corresponds to a physical motion of the gas in hot star atmospheres. This motion may then be connected to wind clumping (Sect. 4.3), since the empirical microturbulent velocities are comparable to the local sound speed at the stellar surface. In order to verify such a picture, multi-dimensional calculations of the $\mathrm{FeCZ}$ and the radiative layers above, including the stellar atmosphere, are required - similar to the recent generation of atmosphere models for cool stars (e.g., Asplund et al. 1999; Wedemeyer et al. 2004).

In Sect. 4.4, we proposed that the FeCZ in hot stars might also produce localized surface magnetic fields, in Galactic stars for luminosities above $\sim 10^{3.2} L_{\odot}$. This could explain the occurrence of DACs (discrete absorption components in UV absorption lines), also in very hot main sequence stars for which pulsational instabilities are not predicted. We further argued that there may be regions of the upper HR diagram for which the presence 
of the FeCZ influences, or even excites, non-radial stellar pulsations (Sect. 4.2).

The FeCZ could also turn out to directly affect the evolution of hot massive stars. If it induces wind clumping, it may alter the stellar wind mass-loss rate. Such a change would also influence the angular momentum loss. In addition magnetic fields produced by the iron convection zone could lead to an enhanced rate of angular momentum loss. These effects become weaker for lower metallicity, where the $\mathrm{FeCZ}$ is less prominent or absent (see Sect. 3).

Finally, we note that the consequences of the FeCZ might be strongest in Wolf-Rayet stars. These stars are so hot that the iron opacity peak, and therefore the $\mathrm{FeCZ}$, can be directly at the stellar surface, or - to be more precise - at the sonic point of the wind flow (Heger \& Langer 1996). This may relate to the very strong clumping found observationally in Wolf-Rayet winds (Lépine \& Moffat 1999; Marchenko et al. 2006), and may be required for an understanding of the very high mass-loss rates of Wolf-Rayet stars (Eichler et al. 1995; Kato \& Iben 1992; Heger \& Langer 1996).

Acknowledgements. The authors would like to thank Myron Smith, Alex Fullerton, Derk Massa and the members of the VLT-FLAMES consortium. M.C. wants to thank the STScI for kind hospitality and the Leids Kerkhoven-Bosscha Fonds for financial support. S.-C. Y. is supported by the DOE SciDAC Program (DOE DE-FC02-06ER41438).

\section{References}

Abbett, W. P., Beaver, M., Davids, B., et al. 1997, ApJ, 480, 395 Aerts, C., Puls, J., Godart, M., \& Dupret, M. 2008, ArXiv e-prints Asplund, M., Nordlund, Å., Trampedach, R., \& Stein, R. F. 1999, A\&A, 346, L17

Asplund, M., Grevesse, N., \& Sauval, A. J. 2005, in Cosmic Abundances as Records of Stellar Evolution and Nucleosynthesis, ed. T. G. Barnes, III, \& F. N. Bash, ASP Conf. Ser., 336, 25

Babel, J., \& Montmerle, T. 1997, ApJ, 485, L29

Badnell, N. R., Bautista, M. A., Butler, K., et al. 2005, MNRAS, 360, 458

Böhm-Vitense, E. 1958, Z. Astrophys., 46, 108

Braithwaite, J., \& Spruit, H. C. 2004, Nature, 431, 819

Charbonneau, P., \& MacGregor, K. B. 2001, ApJ, 559, 1094

Charpinet, S., Fontaine, G., Brassard, P., et al. 1997, ApJ, 483, L123

Cowley, C. R. 1970, The theory of stellar spectra Topics in Astrophysics and

Space Physics (New York: Gordon and Breach)

Cranmer, S. R., \& Owocki, S. P. 1996, ApJ, 462, 469

Davies, B., Oudmaijer, R. D., \& Vink, J. S. 2005, A\&A, 439, 1107

Davies, B., Vink, J. S., \& Oudmaijer, R. D. 2007, A\&A, 469, 1045

Deng, L., \& Xiong, D. R. 2001, MNRAS, 327, 881

Dupret, M. A. 2001, A\&A, 366, 166

Dziembowski, W. A. 2008, ArXiv e-prints

Edmunds, M. G. 1978, A\&A, 64, 103

Eichler, D., Bar Shalom, A., \& Oreg, J. 1995, ApJ, 448, 858

Endal, A. S., \& Sofia, S. 1978, ApJ, 220, 279

Evans, C. J., Smartt, S. J., Lee, J.-K., et al. 2005, A\&A, 437, 467

Eversberg, T., Lepine, S., \& Moffat, A. F. J. 1998, ApJ, 494, 799

Ferrario, L., \& Wickramasinghe, D. T. 2005, MNRAS, 356, 615

Fullerton, A. W., Gies, D. R., \& Bolton, C. T. 1996, ApJS, 103, 475

Fullerton, A. W., Massa, D. L., Prinja, R. K., Owocki, S. P., \& Cranmer, S. R. 1997, A\&A, 327, 699

Gies, D. R., \& Lambert, D. L. 1992, ApJ, 387, 673

Glatzel, W., \& Mehren, S. 1996, MNRAS, 282, 1470

Goldreich, P., \& Kumar, P. 1990, ApJ, 363, 694

Grosdidier, Y., Moffat, A. F. J., Joncas, G., \& Acker, A. 1998, ApJ, 506, L127

Harries, T. J., Howarth, I. D., \& Evans, C. J. 2002, MNRAS, 337, 341

Heger, A., \& Langer, N. 1996, A\&A, 315, 421

Heger, A., Langer, N., \& Woosley, S. E. 2000, ApJ, 528, 368

Heger, A., Woosley, S. E., \& Spruit, H. C. 2005, ApJ, 626, 350

Herrero, A., Kudritzki, R. P., Vilchez, J. M., et al. 1992, A\&A, 261, 209

Hibbins, R. E., Dufton, P. L., Smartt, S. J., \& Rolleston, W. R. J. 1998, A\&A, 332,681
Howarth, I. D., \& Prinja, R. K. 1989, ApJS, 69, 527

Hubeny, I., Heap, S. R., \& Altner, B. 1991, ApJ, 377, L33

Hunter, I., Dufton, P. L., Smartt, S. J., et al. 2007, A\&A, 466, 277

Hunter, I., Brott, I., Lennon, D. J., et al. 2008a, ApJ, 676, L29

Hunter, I., Lennon, D. J., Dufton, P. L., et al. 2008b, A\&A, 479, 541

Iglesias, C. A., \& Rogers, F. J. 1996, ApJ, 464, 943

Iglesias, C. A., Rogers, F. J., \& Wilson, B. G. 1992, ApJ, 397, 717

Kaper, L., Henrichs, H. F., Fullerton, A. W., et al. 1997, A\&A, 327, 281

Kato, M., \& Iben, I. J. 1992, ApJ, 394, 305

Kippenhahn, R., \& Weigert, A. 1990, Stellar Structure and Evolution XVI (Berlin Heidelberg New York: Springer-Verlag), Astron. Astrophys. Library, 468,192

Kiriakidis, M., Fricke, K. J., \& Glatzel, W. 1993, MNRAS, 264, 50

Lefever, K., Puls, J., \& Aerts, C. 2007, A\&A, 463, 1093

Leibacher, J. W., \& Stein, R. F. 1971, Astrophys. Lett., 7, 191

Lennon, D. J., Dufton, P. L., \& Crowley, C. 2003, A\&A, 398, 455

Lépine, S., \& Moffat, A. F. J. 1999, ApJ, 514, 909

Lepine, S., \& Moffat, A. F. J. 2008, ArXiv e-prints, 805

Lighthill, M. F. 1967, in Aerodynamic Phenomena in Stellar Atmospheres, ed.

R. N. Thomas, IAU Symp., 28, 429

Lighthill, M. J. 1952, Royal Soc. London Proc. Ser. A, 211, 564

Lobel, A., \& Blomme, R. 2008, ApJ, 678, 408

Ludwig, H.-G., Freytag, B., \& Steffen, M. 1999, A\&A, 346, 111

Lyubimkov, L. S., Rostopchin, S. I., \& Lambert, D. L. 2004, MNRAS, 351, 745

MacDonald, J., \& Mullan, D. J. 2004, MNRAS, 348, 702

MacGregor, K. B., \& Cassinelli, J. P. 2003, ApJ, 586, 480

Maeder, A., Georgy, C., \& Meynet, G. 2008, A\&A, 479, L37

Marchenko, S. V., Moffat, A. F. J., St-Louis, N., \& Fullerton, A. W. 2006, ApJ, 639, L75

Massa, D., Fullerton, A. W., Nichols, J. S., et al. 1995, ApJ, 452, L53

Massey, P., Puls, J., Pauldrach, A. W. A., et al. 2005, ApJ, 627, 477

Mokiem, M. R., de Koter, A., Evans, C. J., et al. 2007, A\&A, 465, 1003

Morel, T., Butler, K., Aerts, C., Neiner, C., \& Briquet, M. 2006, A\&A, 457, 651

Morel, T., Hubrig, S., \& Briquet, M. 2008, A\&A, 481, 453

Mullan, D. J., \& MacDonald, J. 2005, MNRAS, 356, 1139

Owocki, S. P., \& Puls, J. 1999, ApJ, 510, 355

Pamyatnykh, A. A. 1999, Acta Astron., 49, 119

Parker, E. N. 1975, ApJ, 198, 205

Petrovic, J., Langer, N., Yoon, S.-C., \& Heger, A. 2005, A\&A, 435, 247

Pinsonneault, M. H., Kawaler, S. D., Sofia, S., \& Demarque, P. 1989, ApJ, 338, 424

Prinja, R. K., \& Howarth, I. D. 1988, MNRAS, 233, 123

Prinja, R. K., Massa, D., \& Fullerton, A. W. 2002, A\&A, 388, 587

Puls, J., Markova, N., Scuderi, S., et al. 2006, A\&A, 454, 625

Rolleston, W. R. J., Brown, P. J. F., Dufton, P. L., \& Howarth, I. D. 1996, A\&A, 315,95

Runacres, M. C., \& Owocki, S. P. 2002, A\&A, 381, 1015

Runacres, M. C., \& Owocki, S. P. 2005, A\&A, 429, 323

Saio, H. 1993, MNRAS, 260, 465

Saio, H., Kuschnig, R., Gautschy, A., et al. 2006, ApJ, 650, 1111

Schuessler, M., \& Paehler, A. 1978, A\&A, 68, 57

Shore, S. N. 1992, An introduction to astrophysical hydrodynamics (San Diego: Academic Press)

Smith, K. C., \& Howarth, I. D. 1998, MNRAS, 299, 1146

Spiegel, E. A., \& Weiss, N. O. 1980, Nature, 287, 616

Spiegel, E. A., \& Zahn, J.-P. 1992, A\&A, 265, 106

Spitzer, L. 1962, Physics of Fully Ionized Gases, 2nd edn. (New York: Interscience)

Spruit, H. C. 2002, A\&A, 381, 923

Stankov, A., \& Handler, G. 2005, ApJS, 158, 193

Stein, R. F. 1967, Sol. Phys., 2, 385

Stothers, R. B., \& Chin, C.-W. 1993, ApJ, 408, L85

Townsend, R. H. D., Owocki, S. P., \& Groote, D. 2005, ApJ, 630, L81

Trundle, C., \& Lennon, D. J. 2005, A\&A, 434, 677

Trundle, C., Dufton, P. L., Hunter, I., et al. 2007, A\&A, 471, 625

Ud-Doula, A. \& Owocki, S. P. 2002, ApJ, 576, 413

Ulrich, R. K. 1970, ApJ, 162, 993

van Leeuwen, F., van Genderen, A. M., \& Zegelaar, I. 1998, A\&AS, 128, 117

Vink, J. S., de Koter, A., \& Lamers, H. J. G. L. M. 2001, A\&A, 369, 574

Vrancken, M., Lennon, D. J., Dufton, P. L., \& Lambert, D. L. 2000, A\&A, 358, 639

Wade, G. A., Fullerton, A. W., Donati, J.-F., et al. 2006, A\&A, 451, 195

Wedemeyer, S., Freytag, B., Steffen, M., Ludwig, H.-G., \& Holweger, H. 2004, A\&A, 414, 1121

Yoon, S.-C., Langer, N., \& Norman, C. 2006, A\&A, 460, 199 
M. Cantiello et al.: Sub-surface convection in hot stars, Online Material $p$ I Appendix A: Models 
M. Cantiello et al.: Sub-surface convection in hot stars, Online Material $p 2$

Table A.1. Outermost $4 R_{\odot}$ of a $60 M_{\odot}$ model at solar metallicity $(Z=0.02)$. The table shows physical variables at $t=2.37 \times 10^{6}$ years. Columns contain the progressive grid point of the model, the status ( $R=$ radiative, $C=$ convective), the optical depth $\tau$, the opacity $\varkappa$, the density $\rho$, the radius $R$, the value $M_{*}-M_{r}$ (where $M_{*}$ is the total stellar mass and $M_{r}$ is the mass coordinate), the temperature $T$, the convective velocity $v_{\mathrm{c}}$ and the local sound speed $c_{\mathrm{s}}$. All the values are in cgs units if not otherwise specified.

\begin{tabular}{|c|c|c|c|c|c|c|c|c|c|}
\hline Grid point & Stat & $\tau$ & $x$ & $\rho$ & $R\left[R_{\odot}\right]$ & $M_{*}-M_{r}[\mathrm{~g}]$ & $T[\mathrm{~K}]$ & $v_{\mathrm{c}}\left[\mathrm{km} \mathrm{s}^{-1}\right]$ & $c_{\mathrm{s}}\left[\mathrm{km} \mathrm{s}^{-1}\right]$ \\
\hline 1062 & $\mathrm{R}$ & $1.82 \mathrm{E}+03$ & 0.88736 & $1.2633 \mathrm{E}-08$ & 18.0541 & $2.14 \mathrm{E}-05$ & $2.240 \mathrm{E}+05$ & 0.00 & 267.57 \\
\hline 1063 & $\mathrm{R}$ & $1.76 \mathrm{E}+03$ & 0.89553 & $1.1674 \mathrm{E}-08$ & 18.1381 & $2.06 \mathrm{E}-05$ & $2.214 \mathrm{E}+05$ & 0.00 & 271.52 \\
\hline 1064 & $\mathrm{R}$ & $1.70 \mathrm{E}+03$ & 0.90534 & $1.0813 \mathrm{E}-08$ & 18.2281 & $1.99 \mathrm{E}-05$ & $2.187 \mathrm{E}+05$ & 0.00 & 275.00 \\
\hline 1065 & $\mathrm{R}$ & $1.64 \mathrm{E}+03$ & 0.91747 & $1.0071 \mathrm{E}-08$ & 18.3243 & $1.92 \mathrm{E}-05$ & $2.159 \mathrm{E}+05$ & 0.00 & 277.52 \\
\hline 1066 & $\mathrm{R}$ & $1.57 \mathrm{E}+03$ & 0.92978 & $9.5763 \mathrm{E}-09$ & 18.4264 & $1.84 \mathrm{E}-05$ & $2.136 \mathrm{E}+05$ & 0.00 & 278.41 \\
\hline 1067 & $\mathrm{R}$ & $1.53 \mathrm{E}+03$ & 0.94138 & $9.2743 \mathrm{E}-09$ & 18.4932 & $1.79 \mathrm{E}-05$ & $2.118 \mathrm{E}+05$ & 0.00 & 278.04 \\
\hline 1068 & $\mathrm{C}$ & $1.49 E+03$ & 0.95487 & $9.0395 \mathrm{E}-09$ & 18.5618 & $1.75 \mathrm{E}-05$ & $2.099 \mathrm{E}+05$ & 15.64 & 276.68 \\
\hline 1069 & $\mathrm{C}$ & $1.45 E+03$ & 0.96881 & 8.8184E-09 & 18.6315 & $1.70 \mathrm{E}-05$ & $2.080 \mathrm{E}+05$ & 27.92 & 275.11 \\
\hline 1070 & $\mathrm{C}$ & $1.41 \mathrm{E}+03$ & 0.98203 & $8.6221 \mathrm{E}-09$ & 18.7025 & $1.66 \mathrm{E}-05$ & $2.062 \mathrm{E}+05$ & 34.25 & 273.50 \\
\hline 1071 & $\mathrm{C}$ & $1.37 \mathrm{E}+03$ & 0.99441 & 8.4477E-09 & 18.7644 & $1.62 \mathrm{E}-05$ & $2.046 \mathrm{E}+05$ & 38.62 & 271.88 \\
\hline 1072 & $\mathrm{C}$ & $1.34 \mathrm{E}+03$ & 1.01310 & $8.1959 \mathrm{E}-09$ & 18.8272 & $1.58 \mathrm{E}-05$ & $2.020 \mathrm{E}+05$ & 43.63 & 269.23 \\
\hline 1073 & $\mathrm{C}$ & $1.26 \mathrm{E}+03$ & 1.03152 & 7.9584E-09 & 18.9554 & $1.50 \mathrm{E}-05$ & $1.994 \mathrm{E}+05$ & 47.93 & 266.34 \\
\hline 1074 & $\mathrm{C}$ & $1.23 \mathrm{E}+03$ & 1.04388 & 7.8068E-09 & 19.0207 & $1.46 \mathrm{E}-05$ & $1.977 \mathrm{E}+05$ & 50.34 & 264.25 \\
\hline 1075 & $\mathrm{C}$ & $1.19 \mathrm{E}+03$ & 1.05647 & 7.6607E-09 & 19.0868 & $1.42 \mathrm{E}-05$ & $1.959 \mathrm{E}+05$ & 52.58 & 262.03 \\
\hline 1076 & $\mathrm{C}$ & $1.15 E+03$ & 1.06922 & 7.5199E-09 & 19.1537 & $1.38 \mathrm{E}-05$ & $1.941 \mathrm{E}+05$ & 54.67 & 259.68 \\
\hline 1077 & $\mathrm{C}$ & $1.11 \mathrm{E}+03$ & 1.08107 & 7.3950E-09 & 19.2214 & $1.34 \mathrm{E}-05$ & $1.924 \mathrm{E}+05$ & 56.42 & 257.39 \\
\hline 1078 & $\mathrm{C}$ & $1.08 \mathrm{E}+03$ & 1.09196 & $7.2848 \mathrm{E}-09$ & 19.2792 & $1.30 \mathrm{E}-05$ & $1.908 \mathrm{E}+05$ & 57.92 & 255.19 \\
\hline 1079 & $\mathrm{C}$ & $1.05 \mathrm{E}+03$ & 1.10822 & 7.1261E-09 & 19.3375 & $1.27 \mathrm{E}-05$ & $1.884 \mathrm{E}+05$ & 60.52 & 251.67 \\
\hline 1080 & $\mathrm{C}$ & $9.84 \mathrm{E}+02$ & 1.12405 & 6.9774E-09 & 19.4556 & $1.20 \mathrm{E}-05$ & $1.860 \mathrm{E}+05$ & 62.47 & 247.93 \\
\hline 1081 & $\mathrm{C}$ & $9.52 \mathrm{E}+02$ & 1.13434 & $6.8840 \mathrm{E}-09$ & 19.5154 & 1.17E-05 & $1.843 \mathrm{E}+05$ & 63.23 & 245.26 \\
\hline 1082 & $\mathrm{C}$ & $9.19 \mathrm{E}+02$ & 1.14425 & $6.7952 \mathrm{E}-09$ & 19.5756 & 1.13E-05 & $1.826 \mathrm{E}+05$ & 64.34 & 242.46 \\
\hline 1083 & $\mathrm{C}$ & $8.87 \mathrm{E}+02$ & 1.15367 & $6.7112 \mathrm{E}-09$ & 19.6362 & $1.10 \mathrm{E}-05$ & $1.809 \mathrm{E}+05$ & 65.35 & 239.53 \\
\hline 1084 & $\mathrm{C}$ & $8.54 \mathrm{E}+02$ & 1.16168 & 6.6393E-09 & 19.6972 & $1.07 \mathrm{E}-05$ & $1.793 \mathrm{E}+05$ & 66.06 & 236.74 \\
\hline 1085 & $\mathrm{C}$ & $8.27 \mathrm{E}+02$ & 1.16833 & 6.5784E-09 & 19.7473 & $1.04 \mathrm{E}-05$ & $1.779 \mathrm{E}+05$ & 66.61 & 234.13 \\
\hline 1086 & $\mathrm{C}$ & $8.00 \mathrm{E}+02$ & 1.17736 & 6.4933E-09 & 19.7975 & $1.01 \mathrm{E}-05$ & $1.757 \mathrm{E}+05$ & 68.07 & 230.00 \\
\hline 1087 & $\mathrm{C}$ & $7.47 \mathrm{E}+02$ & 1.18517 & 6.4174E-09 & 19.8986 & $9.56 \mathrm{E}-06$ & $1.734 \mathrm{E}+05$ & 68.74 & 225.65 \\
\hline 1088 & $\mathrm{C}$ & $7.20 \mathrm{E}+02$ & 1.18966 & $6.3727 \mathrm{E}-09$ & 19.9493 & $9.28 \mathrm{E}-06$ & $1.718 \mathrm{E}+05$ & 68.51 & 222.58 \\
\hline 1089 & $\mathrm{C}$ & $6.93 \mathrm{E}+02$ & 1.19323 & $6.3354 \mathrm{E}-09$ & 20.0001 & 9.01E-06 & $1.704 \mathrm{E}+05$ & 68.69 & 219.59 \\
\hline 1090 & $\mathrm{C}$ & $6.70 \mathrm{E}+02$ & 1.19596 & $6.3048 \mathrm{E}-09$ & 20.0443 & 8.77E-06 & $1.690 \mathrm{E}+05$ & 68.77 & 216.70 \\
\hline 1091 & $\mathrm{C}$ & $6.47 \mathrm{E}+02$ & 1.19806 & $6.2785 \mathrm{E}-09$ & 20.0886 & $8.53 \mathrm{E}-06$ & $1.676 \mathrm{E}+05$ & 68.87 & 213.71 \\
\hline 1092 & $\mathrm{C}$ & $6.24 \mathrm{E}+02$ & 1.19947 & $6.2570 \mathrm{E}-09$ & 20.1328 & 8.29E-06 & $1.661 \mathrm{E}+05$ & 68.89 & 210.59 \\
\hline 1093 & $\mathrm{C}$ & $6.01 \mathrm{E}+02$ & 1.20015 & $6.2359 \mathrm{E}-09$ & 20.1770 & $8.05 \mathrm{E}-06$ & $1.641 \mathrm{E}+05$ & 69.26 & 206.20 \\
\hline 1094 & $\mathrm{C}$ & $5.62 \mathrm{E}+02$ & 1.19937 & $6.2257 \mathrm{E}-09$ & 20.2518 & 7.64E-06 & $1.622 \mathrm{E}+05$ & 68.85 & 201.84 \\
\hline 1095 & $\mathrm{C}$ & $5.43 E+02$ & 1.19803 & $6.2251 \mathrm{E}-09$ & 20.2890 & 7.44E-06 & $1.609 \mathrm{E}+05$ & 67.97 & 198.80 \\
\hline 1096 & $\mathrm{C}$ & $5.23 E+02$ & 1.19464 & $6.2343 \mathrm{E}-09$ & 20.3261 & 7.24E-06 & $1.589 \mathrm{E}+05$ & 68.02 & 194.03 \\
\hline 1097 & $\mathrm{C}$ & $4.85 \mathrm{E}+02$ & 1.18825 & $6.2610 \mathrm{E}-09$ & 20.3999 & $6.83 \mathrm{E}-06$ & $1.566 \mathrm{E}+05$ & 67.27 & 188.34 \\
\hline 1098 & $\mathrm{C}$ & $4.59 \mathrm{E}+02$ & 1.18033 & $6.2989 \mathrm{E}-09$ & 20.4506 & $6.55 \mathrm{E}-06$ & $1.546 \mathrm{E}+05$ & 65.73 & 183.40 \\
\hline 1099 & $\mathrm{C}$ & $4.33 \mathrm{E}+02$ & 1.16997 & $6.3530 \mathrm{E}-09$ & 20.5007 & $6.27 \mathrm{E}-06$ & $1.525 \mathrm{E}+05$ & 64.23 & 178.20 \\
\hline 1100 & $\mathrm{C}$ & $4.07 \mathrm{E}+02$ & 1.15723 & $6.4257 \mathrm{E}-09$ & 20.5502 & $5.98 \mathrm{E}-06$ & $1.504 \mathrm{E}+05$ & 62.33 & 172.73 \\
\hline 1101 & $\mathrm{C}$ & $3.82 \mathrm{E}+02$ & 1.14352 & $6.5118 \mathrm{E}-09$ & 20.5989 & $5.70 \mathrm{E}-06$ & $1.483 \mathrm{E}+05$ & 59.95 & 167.44 \\
\hline 1102 & $\mathrm{C}$ & $3.61 \mathrm{E}+02$ & 1.12949 & $6.6089 \mathrm{E}-09$ & 20.6392 & $5.46 \mathrm{E}-06$ & $1.464 \mathrm{E}+05$ & 57.33 & 162.39 \\
\hline 1103 & $\mathrm{C}$ & $3.41 \mathrm{E}+02$ & 1.11461 & $6.7225 \mathrm{E}-09$ & 20.6787 & $5.23 \mathrm{E}-06$ & $1.444 \mathrm{E}+05$ & 54.47 & 157.26 \\
\hline 1104 & $\mathrm{C}$ & $3.21 \mathrm{E}+02$ & 1.09927 & 6.8531E-09 & 20.7158 & $5.00 \mathrm{E}-06$ & $1.424 \mathrm{E}+05$ & 51.18 & 152.06 \\
\hline 1105 & $\mathrm{C}$ & $3.02 \mathrm{E}+02$ & 1.08331 & 7.0035E-09 & 20.7520 & 4.77E-06 & $1.404 \mathrm{E}+05$ & 47.43 & 146.69 \\
\hline 1106 & $\mathrm{C}$ & $2.83 \mathrm{E}+02$ & 1.06508 & 7.1717E-09 & 20.7873 & $4.55 \mathrm{E}-06$ & $1.382 \mathrm{E}+05$ & 43.00 & 141.22 \\
\hline 1107 & $\mathrm{C}$ & $2.65 E+02$ & 1.04501 & 7.3455E-09 & 20.8216 & 4.32E-06 & $1.360 \mathrm{E}+05$ & 37.81 & 135.85 \\
\hline 1108 & $\mathrm{C}$ & $2.48 \mathrm{E}+02$ & 1.02372 & 7.5117E-09 & 20.8535 & 4.10E-06 & $1.338 \mathrm{E}+05$ & 32.08 & 130.70 \\
\hline 1109 & $\mathrm{C}$ & $2.31 \mathrm{E}+02$ & 1.00600 & 7.6295E-09 & 20.8846 & $3.88 \mathrm{E}-06$ & $1.319 \mathrm{E}+05$ & 26.60 & 126.77 \\
\hline 1110 & $\mathrm{C}$ & $2.22 \mathrm{E}+02$ & 0.99256 & $7.7030 \mathrm{E}-09$ & 20.9025 & $3.76 \mathrm{E}-06$ & $1.306 \mathrm{E}+05$ & 22.40 & 123.99 \\
\hline 1111 & $\mathrm{C}$ & $2.13 E+02$ & 0.97901 & $7.7615 \mathrm{E}-09$ & 20.9201 & $3.63 \mathrm{E}-06$ & $1.292 \mathrm{E}+05$ & 19.15 & 121.31 \\
\hline 1112 & $\mathrm{C}$ & $2.03 \mathrm{E}+02$ & 0.96557 & 7.8026E-09 & 20.9376 & $3.50 \mathrm{E}-06$ & $1.277 \mathrm{E}+05$ & 16.16 & 118.76 \\
\hline 1113 & $\mathrm{C}$ & $1.94 \mathrm{E}+02$ & 0.95303 & 7.8243E-09 & 20.9549 & $3.38 \mathrm{E}-06$ & $1.263 \mathrm{E}+05$ & 13.55 & 116.42 \\
\hline 1114 & $\mathrm{C}$ & $1.86 \mathrm{E}+02$ & 0.94136 & 7.8283E-09 & 20.9708 & $3.26 \mathrm{E}-06$ & $1.250 \mathrm{E}+05$ & 11.38 & 114.27 \\
\hline 1115 & $\mathrm{C}$ & $1.78 \mathrm{E}+02$ & 0.92946 & $7.8150 \mathrm{E}-09$ & 20.9866 & $3.15 \mathrm{E}-06$ & $1.236 \mathrm{E}+05$ & 9.54 & 112.21 \\
\hline 1116 & $\mathrm{C}$ & $1.70 \mathrm{E}+02$ & 0.91738 & 7.7833E-09 & 21.0024 & 3.03E-06 & $1.222 \mathrm{E}+05$ & 7.92 & 110.24 \\
\hline 1117 & $\mathrm{C}$ & $1.63 \mathrm{E}+02$ & 0.90353 & 7.7240E-09 & 21.0183 & 2.91E-06 & $1.206 \mathrm{E}+05$ & 6.47 & 108.10 \\
\hline 1118 & $\mathrm{C}$ & $1.53 \mathrm{E}+02$ & 0.88804 & 7.6268E-09 & 21.0386 & $2.77 \mathrm{E}-06$ & $1.187 \mathrm{E}+05$ & 5.08 & 105.83 \\
\hline 1119 & $\mathrm{C}$ & $1.44 \mathrm{E}+02$ & 0.87366 & 7.5046E-09 & 21.0592 & 2.62E-06 & $1.169 \mathrm{E}+05$ & 3.90 & 103.80 \\
\hline 1120 & $\mathrm{C}$ & $1.36 \mathrm{E}+02$ & 0.86054 & 7.3626E-09 & 21.0776 & 2.49E-06 & $1.152 \mathrm{E}+05$ & 3.00 & 102.00 \\
\hline 1121 & $\mathrm{C}$ & $1.28 \mathrm{E}+02$ & 0.84800 & 7.1942E-09 & 21.0964 & $2.36 \mathrm{E}-06$ & $1.134 \mathrm{E}+05$ & 2.30 & 100.28 \\
\hline
\end{tabular}


Table A.1. continued.

\begin{tabular}{|c|c|c|c|c|c|c|c|c|c|}
\hline Grid point & Stat & $\tau$ & $x$ & $\rho$ & $R\left[R_{\odot}\right]$ & $M_{*}-M_{r}[\mathrm{~g}]$ & $T[\mathrm{~K}]$ & $v_{\mathrm{c}}\left[\mathrm{km} \mathrm{s}^{-1}\right]$ & $c_{\mathrm{s}}\left[\mathrm{km} \mathrm{s}^{-1}\right]$ \\
\hline 1122 & $\mathrm{C}$ & $1.20 \mathrm{E}+02$ & 0.83628 & $6.9999 \mathrm{E}-09$ & 21.1155 & $2.22 \mathrm{E}-06$ & $1.115 \mathrm{E}+05$ & 1.75 & 98.63 \\
\hline 1123 & $\mathrm{C}$ & $1.12 \mathrm{E}+02$ & 0.82528 & $6.7743 \mathrm{E}-09$ & 21.1352 & 2.09E-06 & $1.096 \mathrm{E}+05$ & 1.32 & 96.99 \\
\hline 1124 & $\mathrm{C}$ & $1.04 \mathrm{E}+02$ & 0.81748 & $6.5834 \mathrm{E}-09$ & 21.1566 & $1.96 \mathrm{E}-06$ & $1.080 \mathrm{E}+05$ & 1.00 & 95.77 \\
\hline 1125 & $\mathrm{C}$ & $1.00 \mathrm{E}+02$ & 0.81258 & $6.4480 \mathrm{E}-09$ & 21.1676 & $1.89 \mathrm{E}-06$ & $1.069 \mathrm{E}+05$ & 0.80 & 94.97 \\
\hline 1126 & $\mathrm{C}$ & $9.62 \mathrm{E}+01$ & 0.80607 & $6.2478 \mathrm{E}-09$ & 21.1788 & $1.82 \mathrm{E}-06$ & $1.054 \mathrm{E}+05$ & 0.67 & 93.86 \\
\hline 1127 & $\mathrm{C}$ & $8.92 \mathrm{E}+01$ & 0.80011 & $6.0434 \mathrm{E}-09$ & 21.1996 & $1.69 \mathrm{E}-06$ & $1.038 \mathrm{E}+05$ & 0.52 & 92.80 \\
\hline 1128 & $\mathrm{C}$ & $8.57 \mathrm{E}+01$ & 0.79626 & 5.9007E-09 & 21.2104 & $1.63 \mathrm{E}-06$ & $1.028 \mathrm{E}+05$ & 0.42 & 92.09 \\
\hline 1129 & $\mathrm{C}$ & $8.22 \mathrm{E}+01$ & 0.79196 & $5.7334 \mathrm{E}-09$ & 21.2214 & $1.57 \mathrm{E}-06$ & $1.016 \mathrm{E}+05$ & 0.35 & 91.30 \\
\hline 1130 & $\mathrm{C}$ & $7.79 \mathrm{E}+01$ & 0.78715 & $5.5390 \mathrm{E}-09$ & 21.2356 & 1.49E-06 & $1.002 \mathrm{E}+05$ & 0.27 & 90.43 \\
\hline 1131 & $\mathrm{C}$ & $7.36 \mathrm{E}+01$ & 0.78269 & $5.3475 \mathrm{E}-09$ & 21.2503 & $1.41 \mathrm{E}-06$ & $9.880 \mathrm{E}+04$ & 0.21 & 89.60 \\
\hline 1132 & $\mathrm{C}$ & $6.98 \mathrm{E}+01$ & 0.77884 & $5.1603 \mathrm{E}-09$ & 21.2638 & $1.34 \mathrm{E}-06$ & $9.747 \mathrm{E}+04$ & 0.15 & 88.83 \\
\hline 1133 & $\mathrm{C}$ & $6.61 \mathrm{E}+01$ & 0.77531 & $4.9665 \mathrm{E}-09$ & 21.2778 & $1.27 \mathrm{E}-06$ & $9.609 \mathrm{E}+04$ & 0.11 & 88.05 \\
\hline 1134 & $\mathrm{C}$ & $6.23 \mathrm{E}+01$ & 0.77266 & 4.8046E-09 & 21.2923 & $1.20 \mathrm{E}-06$ & $9.494 \mathrm{E}+04$ & 0.07 & 87.42 \\
\hline 1135 & $\mathrm{C}$ & $6.00 \mathrm{E}+01$ & 0.77075 & $4.6775 \mathrm{E}-09$ & 21.3017 & $1.16 \mathrm{E}-06$ & $9.403 \mathrm{E}+04$ & 0.05 & 86.93 \\
\hline 1136 & $\mathrm{C}$ & $5.77 \mathrm{E}+01$ & 0.76804 & $4.4821 \mathrm{E}-09$ & 21.3112 & $1.12 \mathrm{E}-06$ & $9.261 \mathrm{E}+04$ & 0.03 & 86.19 \\
\hline 1137 & $\mathrm{C}$ & $5.32 \mathrm{E}+01$ & 0.76529 & 4.2679E-09 & 21.3311 & $1.03 \mathrm{E}-06$ & $9.105 \mathrm{E}+04$ & 0.01 & 85.39 \\
\hline 1138 & $\mathrm{R}$ & $5.04 \mathrm{E}+01$ & 0.76325 & 4.1031E-09 & 21.3436 & $9.78 \mathrm{E}-07$ & $8.983 \mathrm{E}+04$ & 0.00 & 84.78 \\
\hline 1139 & $\mathrm{R}$ & $4.77 \mathrm{E}+01$ & 0.76129 & 3.9433E-09 & 21.3566 & $9.26 \mathrm{E}-07$ & $8.863 \mathrm{E}+04$ & 0.00 & 84.20 \\
\hline 1140 & $\mathrm{R}$ & $4.53 \mathrm{E}+01$ & 0.75959 & $3.7889 \mathrm{E}-09$ & 21.3687 & $8.80 \mathrm{E}-07$ & $8.746 \mathrm{E}+04$ & 0.00 & 83.64 \\
\hline 1141 & $\mathrm{R}$ & $4.29 \mathrm{E}+01$ & 0.75813 & 3.6339E-09 & 21.3813 & 8.33E-07 & $8.627 \mathrm{E}+04$ & 0.00 & 83.08 \\
\hline 1142 & $\mathrm{R}$ & $4.06 \mathrm{E}+01$ & 0.75688 & $3.4786 \mathrm{E}-09$ & 21.3939 & 7.89E-07 & $8.505 \mathrm{E}+04$ & 0.00 & 82.52 \\
\hline 1143 & $\mathrm{R}$ & $3.83 \mathrm{E}+01$ & 0.75581 & $3.3202 \mathrm{E}-09$ & 21.4071 & 7.44E-07 & $8.378 \mathrm{E}+04$ & 0.00 & 81.95 \\
\hline 1144 & $\mathrm{R}$ & $3.60 \mathrm{E}+01$ & 0.75487 & $3.1587 \mathrm{E}-09$ & 21.4209 & $6.99 \mathrm{E}-07$ & $8.246 \mathrm{E}+04$ & 0.00 & 81.37 \\
\hline 1145 & $\mathrm{R}$ & $3.37 \mathrm{E}+01$ & 0.75414 & 3.0163E-09 & 21.4353 & $6.54 \mathrm{E}-07$ & $8.126 \mathrm{E}+04$ & 0.00 & 80.85 \\
\hline 1146 & $\mathrm{R}$ & $3.20 \mathrm{E}+01$ & 0.75353 & $2.8941 \mathrm{E}-09$ & 21.4464 & $6.22 \mathrm{E}-07$ & $8.021 \mathrm{E}+04$ & 0.00 & 80.40 \\
\hline 1147 & $\mathrm{R}$ & $3.04 \mathrm{E}+01$ & 0.75290 & $2.7700 \mathrm{E}-09$ & 21.4579 & $5.89 \mathrm{E}-07$ & $7.913 \mathrm{E}+04$ & 0.00 & 79.94 \\
\hline 1148 & $\mathrm{R}$ & $2.87 \mathrm{E}+01$ & 0.75225 & $2.6285 \mathrm{E}-09$ & 21.4699 & $5.56 \mathrm{E}-07$ & $7.786 \mathrm{E}+04$ & 0.00 & 79.41 \\
\hline 1149 & $\mathrm{R}$ & $2.67 \mathrm{E}+01$ & 0.75167 & 2.4687E-09 & 21.4856 & $5.16 \mathrm{E}-07$ & $7.638 \mathrm{E}+04$ & 0.00 & 78.80 \\
\hline 1150 & $\mathrm{R}$ & $2.46 \mathrm{E}+01$ & 0.75132 & 2.3386E-09 & 21.5023 & $4.75 \mathrm{E}-07$ & $7.514 \mathrm{E}+04$ & 0.00 & 78.31 \\
\hline 1151 & $\mathrm{R}$ & $2.34 \mathrm{E}+01$ & 0.75114 & $2.2398 \mathrm{E}-09$ & 21.5128 & $4.51 \mathrm{E}-07$ & $7.417 \mathrm{E}+04$ & 0.00 & 77.93 \\
\hline 1152 & $\mathrm{R}$ & $2.22 \mathrm{E}+01$ & 0.75102 & $2.1310 \mathrm{E}-09$ & 21.5238 & 4.27E-07 & $7.308 \mathrm{E}+04$ & 0.00 & 77.51 \\
\hline 1153 & $\mathrm{R}$ & $2.08 \mathrm{E}+01$ & 0.75100 & $2.0117 \mathrm{E}-09$ & 21.5373 & $3.98 \mathrm{E}-07$ & $7.185 \mathrm{E}+04$ & 0.00 & 77.04 \\
\hline 1154 & $\mathrm{R}$ & $1.93 \mathrm{E}+01$ & 0.75107 & $1.9108 \mathrm{E}-09$ & 21.5515 & $3.70 \mathrm{E}-07$ & $7.077 \mathrm{E}+04$ & 0.00 & 76.64 \\
\hline 1155 & $\mathrm{R}$ & $1.84 \mathrm{E}+01$ & 0.75118 & $1.8301 \mathrm{E}-09$ & 21.5615 & $3.51 \mathrm{E}-07$ & $6.989 \mathrm{E}+04$ & 0.00 & 76.32 \\
\hline 1156 & $\mathrm{R}$ & $1.75 \mathrm{E}+01$ & 0.75135 & 1.7497E-09 & 21.5717 & 3.33E-07 & $6.899 \mathrm{E}+04$ & 0.00 & 75.99 \\
\hline 1157 & $\mathrm{R}$ & $1.65 \mathrm{E}+01$ & 0.75159 & $1.6682 \mathrm{E}-09$ & 21.5823 & $3.14 \mathrm{E}-07$ & $6.805 \mathrm{E}+04$ & 0.00 & 75.66 \\
\hline 1158 & $\mathrm{R}$ & $1.56 \mathrm{E}+01$ & 0.75193 & $1.5858 \mathrm{E}-09$ & 21.5934 & 2.96E-07 & $6.707 \mathrm{E}+04$ & 0.00 & 75.32 \\
\hline 1159 & $\mathrm{R}$ & $1.47 \mathrm{E}+01$ & 0.75232 & $1.5107 \mathrm{E}-09$ & 21.6051 & $2.77 \mathrm{E}-07$ & $6.616 \mathrm{E}+04$ & 0.00 & 75.02 \\
\hline 1160 & $\mathrm{R}$ & $1.39 \mathrm{E}+01$ & 0.75277 & $1.4434 \mathrm{E}-09$ & 21.6148 & 2.63E-07 & $6.531 \mathrm{E}+04$ & 0.00 & 74.74 \\
\hline 1161 & $\mathrm{R}$ & $1.32 \mathrm{E}+01$ & 0.75333 & $1.3753 \mathrm{E}-09$ & 21.6250 & $2.48 \mathrm{E}-07$ & $6.444 \mathrm{E}+04$ & 0.00 & 74.45 \\
\hline 1162 & $\mathrm{R}$ & $1.25 \mathrm{E}+01$ & 0.75402 & $1.3065 \mathrm{E}-09$ & 21.6358 & 2.33E-07 & $6.352 \mathrm{E}+04$ & 0.00 & 74.16 \\
\hline 1163 & $\mathrm{R}$ & $1.17 \mathrm{E}+01$ & 0.75502 & $1.2290 \mathrm{E}-09$ & 21.6470 & 2.19E-07 & $6.246 \mathrm{E}+04$ & 0.00 & 73.83 \\
\hline 1164 & $\mathrm{R}$ & $1.09 \mathrm{E}+01$ & 0.75652 & $1.1428 \mathrm{E}-09$ & 21.6617 & $2.01 \mathrm{E}-07$ & $6.122 \mathrm{E}+04$ & 0.00 & 73.45 \\
\hline 1165 & $\mathrm{R}$ & $9.97 \mathrm{E}+00$ & 0.75795 & $1.0761 \mathrm{E}-09$ & 21.6774 & $1.83 \mathrm{E}-07$ & $6.023 \mathrm{E}+04$ & 0.00 & 73.15 \\
\hline 1166 & $\mathrm{R}$ & $9.49 \mathrm{E}+00$ & 0.75910 & $1.0296 \mathrm{E}-09$ & 21.6863 & $1.73 \mathrm{E}-07$ & $5.950 \mathrm{E}+04$ & 0.00 & 72.94 \\
\hline 1167 & $\mathrm{R}$ & $9.01 E+00$ & 0.76037 & $9.8282 \mathrm{E}-10$ & 21.6955 & 1.64E-07 & $5.876 \mathrm{E}+04$ & 0.00 & 72.72 \\
\hline 1168 & $\mathrm{R}$ & $8.54 \mathrm{E}+00$ & 0.76193 & $9.3051 \mathrm{E}-10$ & 21.7052 & $1.54 \mathrm{E}-07$ & $5.789 \mathrm{E}+04$ & 0.00 & 72.46 \\
\hline 1169 & $\mathrm{R}$ & $7.96 \mathrm{E}+00$ & 0.76379 & $8.7258 \mathrm{E}-10$ & 21.7176 & $1.42 \mathrm{E}-07$ & $5.689 \mathrm{E}+04$ & 0.00 & 72.17 \\
\hline 1170 & $\mathrm{R}$ & $7.38 \mathrm{E}+00$ & 0.76562 & $8.1920 \mathrm{E}-10$ & 21.7309 & $1.31 \mathrm{E}-07$ & $5.593 \mathrm{E}+04$ & 0.00 & 71.88 \\
\hline 1171 & $\mathrm{R}$ & $6.89 \mathrm{E}+00$ & 0.76732 & $7.7565 \mathrm{E}-10$ & 21.7426 & $1.21 \mathrm{E}-07$ & $5.511 \mathrm{E}+04$ & 0.00 & 71.64 \\
\hline 1172 & $\mathrm{R}$ & $6.51 \mathrm{E}+00$ & 0.76905 & 7.3709E-10 & 21.7523 & $1.14 \mathrm{E}-07$ & $5.435 \mathrm{E}+04$ & 0.00 & 71.41 \\
\hline 1173 & $\mathrm{R}$ & $6.12 \mathrm{E}+00$ & 0.77070 & 7.0359E-10 & 21.7626 & $1.06 \mathrm{E}-07$ & $5.367 \mathrm{E}+04$ & 0.00 & 71.21 \\
\hline 1174 & $\mathrm{R}$ & $5.84 \mathrm{E}+00$ & 0.77221 & $6.7523 \mathrm{E}-10$ & 21.7704 & $1.00 \mathrm{E}-07$ & $5.308 \mathrm{E}+04$ & 0.00 & 71.03 \\
\hline 1175 & $\mathrm{R}$ & $5.56 \mathrm{E}+00$ & 0.77414 & $6.4136 \mathrm{E}-10$ & 21.7785 & $9.48 \mathrm{E}-08$ & $5.234 \mathrm{E}+04$ & 0.00 & 70.80 \\
\hline 1176 & $\mathrm{R}$ & $5.17 \mathrm{E}+00$ & 0.77624 & $6.0673 \mathrm{E}-10$ & 21.7904 & $8.71 \mathrm{E}-08$ & $5.156 \mathrm{E}+04$ & 0.00 & 70.55 \\
\hline 1177 & $\mathrm{R}$ & $4.87 \mathrm{E}+00$ & 0.77813 & $5.7676 \mathrm{E}-10$ & 21.7999 & $8.13 \mathrm{E}-08$ & $5.085 \mathrm{E}+04$ & 0.00 & 70.32 \\
\hline 1178 & $\mathrm{R}$ & $4.58 \mathrm{E}+00$ & 0.77975 & $5.5149 \mathrm{E}-10$ & 21.8098 & 7.54E-08 & $5.024 \mathrm{E}+04$ & 0.00 & 70.11 \\
\hline 1179 & $\mathrm{R}$ & $4.37 \mathrm{E}+00$ & 0.78112 & $5.3095 \mathrm{E}-10$ & 21.8169 & 7.15E-08 & $4.972 \mathrm{E}+04$ & 0.00 & 69.94 \\
\hline 1180 & $\mathrm{R}$ & $4.17 \mathrm{E}+00$ & 0.78266 & $5.1037 \mathrm{E}-10$ & 21.8243 & $6.75 \mathrm{E}-08$ & $4.918 \mathrm{E}+04$ & 0.00 & 69.75 \\
\hline 1181 & $\mathrm{R}$ & $3.96 \mathrm{E}+00$ & 0.78445 & $4.8907 \mathrm{E}-10$ & 21.8320 & $6.35 \mathrm{E}-08$ & $4.861 \mathrm{E}+04$ & 0.00 & 69.55 \\
\hline 1182 & $\mathrm{R}$ & $3.74 \mathrm{E}+00$ & 0.78647 & $4.6708 \mathrm{E}-10$ & 21.8405 & $5.93 \mathrm{E}-08$ & $4.799 \mathrm{E}+04$ & 0.00 & 69.32 \\
\hline 1183 & $\mathrm{R}$ & $3.53 \mathrm{E}+00$ & 0.78864 & $4.4509 \mathrm{E}-10$ & 21.8494 & $5.51 \mathrm{E}-08$ & $4.735 E+04$ & 0.00 & 69.07 \\
\hline 1184 & $\mathrm{R}$ & $3.31 \mathrm{E}+00$ & 0.79092 & $4.2311 \mathrm{E}-10$ & 21.8587 & $5.08 \mathrm{E}-08$ & $4.669 \mathrm{E}+04$ & 0.00 & 68.80 \\
\hline
\end{tabular}


M. Cantiello et al.: Sub-surface convection in hot stars, Online Material p 4

Table A.1. continued.

\begin{tabular}{|c|c|c|c|c|c|c|c|c|c|}
\hline Grid point & Stat & $\tau$ & $x$ & $\rho$ & $R\left[R_{\odot}\right]$ & $M_{*}-M_{r}[\mathrm{~g}]$ & $T[\mathrm{~K}]$ & $v_{\mathrm{c}}\left[\mathrm{km} \mathrm{s}^{-1}\right]$ & $c_{\mathrm{s}}\left[\mathrm{km} \mathrm{s}^{-1}\right]$ \\
\hline 1185 & $\overline{\mathrm{R}}$ & $3.09 \mathrm{E}+00$ & 0.79285 & $4.0457 \mathrm{E}-10$ & 21.8686 & $4.66 \mathrm{E}-08$ & $4.610 \mathrm{E}+04$ & 0.00 & 68.55 \\
\hline 1186 & $\mathrm{R}$ & $2.94 \mathrm{E}+00$ & 0.79437 & $3.8946 \mathrm{E}-10$ & 21.8756 & $4.37 \mathrm{E}-08$ & $4.560 \mathrm{E}+04$ & 0.00 & 68.33 \\
\hline 1187 & $\mathrm{R}$ & $2.78 \mathrm{E}+00$ & 0.79580 & $3.7432 \mathrm{E}-10$ & 21.8830 & $4.08 \mathrm{E}-08$ & $4.509 E+04$ & 0.00 & 68.10 \\
\hline 1188 & $\mathrm{R}$ & $2.63 \mathrm{E}+00$ & 0.79704 & $3.5915 \mathrm{E}-10$ & 21.8906 & $3.79 \mathrm{E}-08$ & $4.456 \mathrm{E}+04$ & 0.00 & 67.85 \\
\hline 1189 & $\mathrm{R}$ & $2.48 \mathrm{E}+00$ & 0.79808 & $3.4600 \mathrm{E}-10$ & 21.8985 & $3.49 \mathrm{E}-08$ & $4.409 E+04$ & 0.00 & 67.62 \\
\hline 1190 & $\mathrm{R}$ & $2.37 \mathrm{E}+00$ & 0.79898 & $3.3489 \mathrm{E}-10$ & 21.9045 & $3.28 \mathrm{E}-08$ & $4.367 \mathrm{E}+04$ & 0.00 & 67.42 \\
\hline 1191 & $\mathrm{R}$ & $2.26 \mathrm{E}+00$ & 0.80002 & $3.2165 \mathrm{E}-10$ & 21.9107 & $3.07 \mathrm{E}-08$ & $4.317 \mathrm{E}+04$ & 0.00 & 67.16 \\
\hline 1192 & $\mathrm{R}$ & $2.10 \mathrm{E}+00$ & 0.80087 & $3.0983 \mathrm{E}-10$ & 21.9195 & $2.78 \mathrm{E}-08$ & $4.271 \mathrm{E}+04$ & 0.00 & 66.93 \\
\hline 1193 & $\mathrm{R}$ & $2.02 \mathrm{E}+00$ & 0.80139 & $3.0157 \mathrm{E}-10$ & 21.9244 & $2.62 \mathrm{E}-08$ & $4.238 \mathrm{E}+04$ & 0.00 & 66.76 \\
\hline 1194 & $\mathrm{R}$ & $1.94 \mathrm{E}+00$ & 0.80182 & $2.9326 \mathrm{E}-10$ & 21.9295 & $2.47 \mathrm{E}-08$ & $4.204 \mathrm{E}+04$ & 0.00 & 66.58 \\
\hline 1195 & $\mathrm{R}$ & $1.86 \mathrm{E}+00$ & 0.80213 & $2.8491 \mathrm{E}-10$ & 21.9346 & $2.31 \mathrm{E}-08$ & $4.169 E+04$ & 0.00 & 66.40 \\
\hline 1196 & $\mathrm{R}$ & $1.78 \mathrm{E}+00$ & 0.80227 & $2.7606 \mathrm{E}-10$ & 21.9400 & $2.16 \mathrm{E}-08$ & $4.131 \mathrm{E}+04$ & 0.00 & 66.20 \\
\hline 1197 & $\mathrm{R}$ & $1.69 \mathrm{E}+00$ & 0.80215 & $2.6667 \mathrm{E}-10$ & 21.9460 & $1.99 \mathrm{E}-08$ & $4.091 \mathrm{E}+04$ & 0.00 & 65.99 \\
\hline 1198 & $\mathrm{R}$ & $1.59 \mathrm{E}+00$ & 0.80180 & $2.5928 \mathrm{E}-10$ & 21.9523 & $1.81 \mathrm{E}-08$ & $4.058 \mathrm{E}+04$ & 0.00 & 65.83 \\
\hline 1199 & $\mathrm{R}$ & $1.54 \mathrm{E}+00$ & 0.80112 & $2.5125 \mathrm{E}-10$ & 21.9559 & $1.72 \mathrm{E}-08$ & $4.023 \mathrm{E}+04$ & 0.00 & 65.65 \\
\hline 1200 & $\mathrm{R}$ & $1.44 \mathrm{E}+00$ & 0.80003 & $2.4310 \mathrm{E}-10$ & 21.9634 & $1.53 \mathrm{E}-08$ & $3.986 \mathrm{E}+04$ & 0.00 & 65.48 \\
\hline 1201 & $\mathrm{R}$ & $1.39 \mathrm{E}+00$ & 0.79900 & $2.3729 \mathrm{E}-10$ & 21.9672 & $1.43 \mathrm{E}-08$ & $3.960 \mathrm{E}+04$ & 0.00 & 65.36 \\
\hline 1202 & $\mathrm{R}$ & $1.34 \mathrm{E}+00$ & 0.79775 & $2.3130 \mathrm{E}-10$ & 21.9715 & $1.32 \mathrm{E}-08$ & $3.933 E+04$ & 0.00 & 65.25 \\
\hline 1203 & $\mathrm{R}$ & $1.29 \mathrm{E}+00$ & 0.79556 & $2.2262 \mathrm{E}-10$ & 21.9757 & $1.22 \mathrm{E}-08$ & $3.894 \mathrm{E}+04$ & 0.00 & 65.10 \\
\hline 1204 & $\mathrm{R}$ & $1.19 \mathrm{E}+00$ & 0.79285 & $2.1391 \mathrm{E}-10$ & 21.9841 & 1.03E-08 & $3.854 \mathrm{E}+04$ & 0.00 & 64.98 \\
\hline 1205 & $\mathrm{R}$ & $1.14 \mathrm{E}+00$ & 0.78970 & $2.0556 \mathrm{E}-10$ & 21.9883 & 9.40E-09 & $3.817 \mathrm{E}+04$ & 0.00 & 64.90 \\
\hline 1206 & $\mathrm{R}$ & $1.05 \mathrm{E}+00$ & 0.78603 & $1.9736 \mathrm{E}-10$ & 21.9964 & 7.68E-09 & $3.780 \mathrm{E}+04$ & 0.00 & 64.86 \\
\hline 1207 & $\mathrm{R}$ & $1.01 \mathrm{E}+00$ & 0.78257 & $1.9068 \mathrm{E}-10$ & 22.0003 & $6.88 \mathrm{E}-09$ & $3.751 \mathrm{E}+04$ & 0.00 & 64.86 \\
\hline 1208 & $\mathrm{R}$ & $9.50 \mathrm{E}-01$ & 0.77882 & $1.8424 \mathrm{E}-10$ & 22.0064 & 5.69E-09 & $3.723 E+04$ & 0.00 & 64.89 \\
\hline 1209 & $\mathrm{R}$ & $9.17 \mathrm{E}-01$ & 0.77546 & $1.7903 \mathrm{E}-10$ & 22.0098 & 5.03E-09 & $3.700 \mathrm{E}+04$ & 0.00 & 64.95 \\
\hline 1210 & $\mathrm{R}$ & $8.76 \mathrm{E}-01$ & 0.77201 & $1.7411 \mathrm{E}-10$ & 22.0141 & 4.24E-09 & $3.680 \mathrm{E}+04$ & 0.00 & 65.03 \\
\hline 1211 & $\mathrm{R}$ & 8.49E-01 & 0.76795 & $1.6877 \mathrm{E}-10$ & 22.0172 & 3.69E-09 & $3.658 \mathrm{E}+04$ & 0.00 & 65.15 \\
\hline 1212 & $\mathrm{R}$ & 8.06E-01 & 0.76388 & $1.6380 \mathrm{E}-10$ & 22.0221 & 2.83E-09 & $3.637 \mathrm{E}+04$ & 0.00 & 65.30 \\
\hline 1213 & $\mathrm{R}$ & 7.85E-01 & 0.76046 & $1.5988 \mathrm{E}-10$ & 22.0245 & 2.42E-09 & $3.622 \mathrm{E}+04$ & 0.00 & 65.44 \\
\hline 1214 & $\mathrm{R}$ & 7.57E-01 & 0.75691 & $1.5601 \mathrm{E}-10$ & 22.0280 & $1.85 \mathrm{E}-09$ & $3.606 \mathrm{E}+04$ & 0.00 & 65.60 \\
\hline 1215 & $\mathrm{R}$ & 7.39E-01 & 0.75246 & $1.5144 \mathrm{E}-10$ & 22.0303 & 1.47E-09 & $3.589 \mathrm{E}+04$ & 0.00 & 65.83 \\
\hline 1216 & $\mathrm{R}$ & 7.04E-01 & 0.74810 & $1.4719 \mathrm{E}-10$ & 22.0348 & 7.64E-10 & $3.573 \mathrm{E}+04$ & 0.00 & 66.07 \\
\hline 1217 & $\mathrm{R}$ & $6.91 \mathrm{E}-01$ & 0.74443 & $1.4378 \mathrm{E}-10$ & 22.0366 & $4.96 \mathrm{E}-10$ & $3.560 \mathrm{E}+04$ & 0.00 & 66.29 \\
\hline 1218 & $\mathrm{R}$ & $6.67 \mathrm{E}-01$ & 0.74192 & $1.4152 \mathrm{E}-10$ & 22.0399 & $0.00 \mathrm{E}+00$ & $3.552 \mathrm{E}+04$ & 0.00 & 66.45 \\
\hline
\end{tabular}


Table A.2. Outermost $1 R_{\odot}$ of a $20 M_{\odot}$ model at solar metallicity $(Z=0.02)$. The table shows the physical variables at $t=6.41 \times 10^{6}$ years. Columns contain the progressive grid point of the model, the status ( $R=$ radiative, $C=$ convective), the optical depth $\tau$, the opacity $\varkappa$, the density $\rho$, the radius $R$, the value $M_{*}-M_{r}$ (where $M_{*}$ is the total stellar mass and $M_{r}$ is the mass coordinate), the temperature $T$, the convective velocity $v_{\mathrm{c}}$ and the local sound speed $c_{\mathrm{s}}$. All the values are in $\operatorname{cgs}$ units if not otherwise specified.

\begin{tabular}{|c|c|c|c|c|c|c|c|c|c|}
\hline Grid point & Stat & $\bar{\tau}$ & $\bar{x}$ & $\overline{\rho \rho}$ & $R\left[R_{\odot}\right]$ & $M_{*}-M_{r}[\mathrm{~g}]$ & $\overline{T[\mathrm{~K}]}$ & $\overline{v_{\mathrm{c}}\left[\mathrm{km} \mathrm{s}^{-1}\right]}$ & $\bar{c} c_{\mathrm{s}}\left[\mathrm{km} \mathrm{s}^{-1}\right]$ \\
\hline 1009 & $\overline{\mathrm{R}}$ & $1.88 \mathrm{E}+04$ & 1.15006 & $9.1148 \mathrm{E}-07$ & 9.4516 & $3.91 \mathrm{E}-05$ & $3.415 \mathrm{E}+05$ & 0.00 & 110.14 \\
\hline 1010 & $\mathrm{R}$ & $1.80 \mathrm{E}+04$ & 1.16843 & $8.4671 \mathrm{E}-07$ & 9.4634 & $3.70 \mathrm{E}-05$ & $3.363 \mathrm{E}+05$ & 0.00 & 109.93 \\
\hline 1011 & $\mathrm{R}$ & $1.68 \mathrm{E}+04$ & 1.18658 & 7.8819E-07 & 9.4826 & 3.39E-05 & $3.313 \mathrm{E}+05$ & 0.00 & 109.74 \\
\hline 1012 & $\mathrm{R}$ & $1.61 \mathrm{E}+04$ & 1.19963 & 7.4912E-07 & 9.4929 & $3.23 \mathrm{E}-05$ & $3.279 \mathrm{E}+05$ & 0.00 & 109.61 \\
\hline 1013 & $\mathrm{R}$ & $1.55 \mathrm{E}+04$ & 1.21788 & $6.9847 \mathrm{E}-07$ & 9.5037 & $3.08 \mathrm{E}-05$ & $3.232 \mathrm{E}+05$ & 0.00 & 109.45 \\
\hline 1014 & $\mathrm{R}$ & $1.45 \mathrm{E}+04$ & 1.24209 & $6.3760 \mathrm{E}-07$ & 9.5220 & $2.83 \mathrm{E}-05$ & $3.172 \mathrm{E}+05$ & 0.00 & 109.26 \\
\hline 1015 & $\mathrm{R}$ & $1.35 \mathrm{E}+04$ & 1.26170 & $5.9299 \mathrm{E}-07$ & 9.5413 & $2.59 \mathrm{E}-05$ & $3.125 \mathrm{E}+05$ & 0.00 & 109.14 \\
\hline 1016 & $\mathrm{R}$ & $1.30 \mathrm{E}+04$ & 1.27584 & $5.6324 \mathrm{E}-07$ & 9.5516 & $2.47 \mathrm{E}-05$ & $3.093 \mathrm{E}+05$ & 0.00 & 109.06 \\
\hline 1017 & $\mathrm{R}$ & $1.24 \mathrm{E}+04$ & 1.29061 & $5.3416 \mathrm{E}-07$ & 9.5624 & $2.36 \mathrm{E}-05$ & $3.060 \mathrm{E}+05$ & 0.00 & 108.98 \\
\hline 1018 & $\mathrm{R}$ & $1.19 \mathrm{E}+04$ & 1.30554 & $5.0665 \mathrm{E}-07$ & 9.5733 & 2.24E-05 & $3.027 \mathrm{E}+05$ & 0.00 & 108.92 \\
\hline 1019 & $\mathrm{R}$ & $1.15 \mathrm{E}+04$ & 1.32091 & 4.8003E-07 & 9.5841 & 2.14E-05 & $2.994 \mathrm{E}+05$ & 0.00 & 108.86 \\
\hline 1020 & $\mathrm{R}$ & $1.10 \mathrm{E}+04$ & 1.33683 & $4.5439 \mathrm{E}-07$ & 9.5954 & $2.03 \mathrm{E}-05$ & $2.961 \mathrm{E}+05$ & 0.00 & 108.81 \\
\hline 1021 & $\mathrm{R}$ & $1.05 \mathrm{E}+04$ & 1.35328 & $4.2968 \mathrm{E}-07$ & 9.6065 & $1.93 \mathrm{E}-05$ & $2.928 \mathrm{E}+05$ & 0.00 & 108.77 \\
\hline 1022 & $\mathrm{R}$ & $1.01 \mathrm{E}+04$ & 1.37094 & 4.0503E-07 & 9.6182 & $1.83 \mathrm{E}-05$ & $2.893 \mathrm{E}+05$ & 0.00 & 108.73 \\
\hline 1023 & $\mathrm{R}$ & $9.62 \mathrm{E}+03$ & 1.38833 & $3.8248 \mathrm{E}-07$ & 9.6306 & $1.73 \mathrm{E}-05$ & $2.860 \mathrm{E}+05$ & 0.00 & 108.71 \\
\hline 1024 & $\mathrm{R}$ & $9.24 \mathrm{E}+03$ & 1.40532 & $3.6200 \mathrm{E}-07$ & 9.6416 & $1.65 \mathrm{E}-05$ & $2.828 \mathrm{E}+05$ & 0.00 & 108.69 \\
\hline 1025 & $\mathrm{R}$ & $8.85 \mathrm{E}+03$ & 1.42367 & 3.4159E-07 & 9.6531 & $1.57 \mathrm{E}-05$ & $2.795 \mathrm{E}+05$ & 0.00 & 108.68 \\
\hline 1026 & $\mathrm{R}$ & $8.45 \mathrm{E}+03$ & 1.44389 & $3.2125 \mathrm{E}-07$ & 9.6653 & $1.48 \mathrm{E}-05$ & $2.761 \mathrm{E}+05$ & 0.00 & 108.67 \\
\hline 1027 & $\mathrm{R}$ & $8.05 E+03$ & 1.46417 & $3.0276 \mathrm{E}-07$ & 9.6783 & $1.40 \mathrm{E}-05$ & $2.728 \mathrm{E}+05$ & 0.00 & 108.67 \\
\hline 1028 & $\mathrm{R}$ & $7.72 \mathrm{E}+03$ & 1.48419 & $2.8611 \mathrm{E}-07$ & 9.6896 & 1.33E-05 & $2.697 \mathrm{E}+05$ & 0.00 & 108.68 \\
\hline 1029 & $\mathrm{R}$ & $7.38 \mathrm{E}+03$ & 1.50595 & 2.6954E-07 & 9.7016 & $1.26 \mathrm{E}-05$ & $2.664 \mathrm{E}+05$ & 0.00 & 108.68 \\
\hline 1030 & $\mathrm{R}$ & $7.04 \mathrm{E}+03$ & 1.52815 & $2.5408 \mathrm{E}-07$ & 9.7142 & 1.19E-05 & $2.632 \mathrm{E}+05$ & 0.00 & 108.68 \\
\hline 1031 & $\mathrm{R}$ & $6.73 \mathrm{E}+03$ & 1.55060 & 2.3969E-07 & 9.7260 & $1.13 \mathrm{E}-05$ & $2.601 \mathrm{E}+05$ & 0.00 & 108.68 \\
\hline 1032 & $\mathrm{R}$ & $6.42 \mathrm{E}+03$ & 1.57310 & 2.2639E-07 & 9.7385 & $1.07 \mathrm{E}-05$ & $2.570 \mathrm{E}+05$ & 0.00 & 108.67 \\
\hline 1033 & $\mathrm{R}$ & $6.15 E+03$ & 1.59542 & $2.1415 \mathrm{E}-07$ & 9.7499 & $1.02 \mathrm{E}-05$ & $2.541 \mathrm{E}+05$ & 0.00 & 108.66 \\
\hline 1034 & $\mathrm{R}$ & $5.88 \mathrm{E}+03$ & 1.62281 & $2.0030 \mathrm{E}-07$ & 9.7619 & 9.69E-06 & $2.505 \mathrm{E}+05$ & 0.00 & 108.63 \\
\hline 1035 & $\mathrm{R}$ & $5.53 \mathrm{E}+03$ & 1.65803 & 1.8493E-07 & 9.7784 & $9.02 \mathrm{E}-06$ & $2.463 \mathrm{E}+05$ & 0.00 & 108.57 \\
\hline 1036 & $\mathrm{R}$ & $5.17 \mathrm{E}+03$ & 1.69089 & $1.7276 \mathrm{E}-07$ & 9.7961 & 8.35E-06 & $2.426 \mathrm{E}+05$ & 0.00 & 108.48 \\
\hline 1037 & $\mathrm{R}$ & $4.94 \mathrm{E}+03$ & 1.72391 & $1.6211 \mathrm{E}-07$ & 9.8077 & 7.94E-06 & $2.392 \mathrm{E}+05$ & 0.00 & 108.36 \\
\hline 1038 & $\mathrm{R}$ & $4.64 \mathrm{E}+03$ & 1.76662 & $1.5016 \mathrm{E}-07$ & 9.8242 & 7.39E-06 & $2.350 \mathrm{E}+05$ & 0.00 & 108.14 \\
\hline 1039 & $\mathrm{R}$ & $4.32 \mathrm{E}+03$ & 1.80536 & $1.4067 \mathrm{E}-07$ & 9.8420 & 6.84E-06 & $2.314 \mathrm{E}+05$ & 0.00 & 107.88 \\
\hline 1040 & $\mathrm{R}$ & $4.12 \mathrm{E}+03$ & 1.83771 & $1.3352 \mathrm{E}-07$ & 9.8539 & $6.50 \mathrm{E}-06$ & $2.284 \mathrm{E}+05$ & 0.00 & 107.60 \\
\hline 1041 & $\mathrm{R}$ & $3.91 \mathrm{E}+03$ & 1.86954 & $1.2702 \mathrm{E}-07$ & 9.8664 & $6.15 \mathrm{E}-06$ & $2.255 \mathrm{E}+05$ & 0.00 & 107.28 \\
\hline 1042 & $\mathrm{R}$ & $3.73 \mathrm{E}+03$ & 1.90044 & $1.2115 \mathrm{E}-07$ & 9.8776 & $5.85 \mathrm{E}-06$ & $2.226 \mathrm{E}+05$ & 0.00 & 106.90 \\
\hline 1043 & $\mathrm{R}$ & $3.55 \mathrm{E}+03$ & 1.93471 & $1.1542 \mathrm{E}-07$ & 9.8894 & $5.56 \mathrm{E}-06$ & $2.197 \mathrm{E}+05$ & 0.00 & 106.44 \\
\hline 1044 & $\mathrm{R}$ & $3.37 \mathrm{E}+03$ & 1.97305 & $1.0986 \mathrm{E}-07$ & 9.9017 & $5.26 \mathrm{E}-06$ & $2.165 \mathrm{E}+05$ & 0.00 & 105.86 \\
\hline 1045 & $\mathrm{R}$ & $3.18 \mathrm{E}+03$ & 2.01222 & $1.0492 \mathrm{E}-07$ & 9.9146 & $4.96 \mathrm{E}-06$ & $2.134 \mathrm{E}+05$ & 0.00 & 105.21 \\
\hline 1046 & $\mathrm{R}$ & $3.01 \mathrm{E}+03$ & 2.05131 & $1.0056 \mathrm{E}-07$ & 9.9260 & 4.71E-06 & $2.105 \mathrm{E}+05$ & 0.00 & 104.47 \\
\hline 1047 & $\mathrm{C}$ & $2.85 \mathrm{E}+03$ & 2.09321 & $9.6390 \mathrm{E}-08$ & 9.9379 & $4.46 \mathrm{E}-06$ & $2.073 \mathrm{E}+05$ & 0.27 & 103.59 \\
\hline 1048 & $\mathrm{C}$ & $2.68 \mathrm{E}+03$ & 2.13709 & $9.2418 \mathrm{E}-08$ & 9.9502 & 4.21E-06 & $2.039 \mathrm{E}+05$ & 3.48 & 102.53 \\
\hline 1049 & $\mathrm{C}$ & $2.50 \mathrm{E}+03$ & 2.17706 & 8.8976E-08 & 9.9630 & $3.96 \mathrm{E}-06$ & $2.007 \mathrm{E}+05$ & 5.74 & 101.40 \\
\hline 1050 & $\mathrm{C}$ & $2.36 \mathrm{E}+03$ & 2.21180 & 8.6007E-08 & 9.9739 & $3.76 \mathrm{E}-06$ & $1.976 \mathrm{E}+05$ & 7.30 & 100.24 \\
\hline 1051 & $\mathrm{C}$ & $2.21 \mathrm{E}+03$ & 2.24604 & $8.3153 \mathrm{E}-08$ & 9.9851 & $3.55 \mathrm{E}-06$ & $1.943 \mathrm{E}+05$ & 8.51 & 98.93 \\
\hline 1052 & $\mathrm{C}$ & $2.07 \mathrm{E}+03$ & 2.27681 & $8.0595 \mathrm{E}-08$ & 9.9967 & $3.35 \mathrm{E}-06$ & $1.911 \mathrm{E}+05$ & 9.43 & 97.55 \\
\hline 1053 & $\mathrm{C}$ & $1.94 \mathrm{E}+03$ & 2.30324 & 7.8309E-08 & 10.0070 & $3.17 \mathrm{E}-06$ & $1.879 \mathrm{E}+05$ & 10.10 & 96.14 \\
\hline 1054 & $\mathrm{C}$ & $1.80 \mathrm{E}+03$ & 2.32459 & 7.6279E-08 & 10.0177 & 2.99E-06 & $1.848 \mathrm{E}+05$ & 10.53 & 94.69 \\
\hline 1055 & $\mathrm{C}$ & $1.69 \mathrm{E}+03$ & 2.34028 & 7.4483E-08 & 10.0269 & 2.84E-06 & $1.818 \mathrm{E}+05$ & 10.74 & 93.24 \\
\hline 1056 & $\mathrm{C}$ & $1.58 \mathrm{E}+03$ & 2.35064 & 7.2749E-08 & 10.0363 & 2.69E-06 & $1.786 \mathrm{E}+05$ & 10.81 & 91.67 \\
\hline 1057 & $\mathrm{C}$ & $1.47 \mathrm{E}+03$ & 2.35334 & 7.1321E-08 & 10.0460 & 2.54E-06 & $1.758 \mathrm{E}+05$ & 10.61 & 90.24 \\
\hline 1058 & $\mathrm{C}$ & $1.39 \mathrm{E}+03$ & 2.34958 & 7.0171E-08 & 10.0528 & $2.43 \mathrm{E}-06$ & $1.734 \mathrm{E}+05$ & 10.26 & 89.00 \\
\hline 1059 & $\mathrm{C}$ & $1.31 \mathrm{E}+03$ & 2.33995 & $6.9027 \mathrm{E}-08$ & 10.0598 & 2.33E-06 & $1.708 \mathrm{E}+05$ & 9.87 & 87.70 \\
\hline 1060 & $\mathrm{C}$ & $1.23 \mathrm{E}+03$ & 2.32417 & $6.7878 \mathrm{E}-08$ & 10.0668 & 2.22E-06 & $1.682 \mathrm{E}+05$ & 9.34 & 86.34 \\
\hline 1061 & $\mathrm{C}$ & $1.16 \mathrm{E}+03$ & 2.30414 & 6.6802E-08 & 10.0740 & $2.12 \mathrm{E}-06$ & $1.657 \mathrm{E}+05$ & 8.69 & 85.05 \\
\hline 1062 & $\mathrm{C}$ & $1.09 \mathrm{E}+03$ & 2.28134 & 6.5791E-08 & 10.0801 & 2.03E-06 & $1.633 \mathrm{E}+05$ & 8.00 & 83.83 \\
\hline 1063 & $\mathrm{C}$ & $1.03 \mathrm{E}+03$ & 2.25516 & $6.4766 \mathrm{E}-08$ & 10.0863 & $1.94 \mathrm{E}-06$ & $1.610 \mathrm{E}+05$ & 7.32 & 82.61 \\
\hline 1064 & $\mathrm{C}$ & $9.71 \mathrm{E}+02$ & 2.22633 & $6.3719 \mathrm{E}-08$ & 10.0923 & $1.86 \mathrm{E}-06$ & $1.585 \mathrm{E}+05$ & 6.62 & 81.39 \\
\hline 1065 & $\mathrm{C}$ & $9.12 \mathrm{E}+02$ & 2.19204 & $6.2614 \mathrm{E}-08$ & 10.0984 & $1.77 \mathrm{E}-06$ & $1.561 \mathrm{E}+05$ & 5.91 & 80.16 \\
\hline 1066 & $\mathrm{C}$ & $8.55 \mathrm{E}+02$ & 2.15002 & $6.1433 \mathrm{E}-08$ & 10.1046 & 1.69E-06 & $1.535 \mathrm{E}+05$ & 5.16 & 78.90 \\
\hline 1067 & $\mathrm{C}$ & $8.00 \mathrm{E}+02$ & 2.10271 & 6.0183E-08 & 10.1109 & $1.60 \mathrm{E}-06$ & $1.509 \mathrm{E}+05$ & 4.39 & 77.68 \\
\hline 1068 & $\mathrm{C}$ & $7.48 \mathrm{E}+02$ & 2.05208 & 5.8853E-08 & 10.1170 & $1.52 \mathrm{E}-06$ & $1.483 \mathrm{E}+05$ & 3.66 & 76.48 \\
\hline 1069 & $\mathrm{C}$ & $6.97 \mathrm{E}+02$ & 2.01013 & $5.7714 \mathrm{E}-08$ & 10.1233 & $1.44 \mathrm{E}-06$ & $1.462 \mathrm{E}+05$ & 3.03 & 75.53 \\
\hline 1070 & $\mathrm{C}$ & $6.68 \mathrm{E}+02$ & 1.97885 & $5.6821 \mathrm{E}-08$ & 10.1270 & $1.40 \mathrm{E}-06$ & $1.447 \mathrm{E}+05$ & 2.59 & 74.84 \\
\hline
\end{tabular}


Table A.2. continued.

\begin{tabular}{|c|c|c|c|c|c|c|c|c|c|}
\hline Grid point & Stat & $\tau$ & $x$ & $\rho$ & 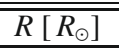 & $\overline{M_{*}-M_{r}[\mathrm{~g}]}$ & $\overline{T[\mathrm{~K}]}$ & $\bar{v}_{\mathrm{c}}\left[\mathrm{km} \mathrm{s}^{-1}\right]$ & $\bar{c}_{c_{\mathrm{s}}\left[\mathrm{km} \mathrm{s}^{-1}\right]}$ \\
\hline 1071 & $\mathrm{C}$ & $6.40 \mathrm{E}+02$ & 1.94778 & $5.5882 \mathrm{E}-08$ & 10.1308 & $1.35 \mathrm{E}-06$ & $1.431 \mathrm{E}+05$ & 2.26 & 74.14 \\
\hline 1072 & $\mathrm{C}$ & $6.11 \mathrm{E}+02$ & 1.91749 & $5.4897 \mathrm{E}-08$ & 10.1346 & $1.30 \mathrm{E}-06$ & $1.415 \mathrm{E}+05$ & 1.97 & 73.45 \\
\hline 1073 & $\mathrm{C}$ & $5.84 \mathrm{E}+02$ & 1.88866 & $5.3907 \mathrm{E}-08$ & 10.1386 & $1.25 \mathrm{E}-06$ & $1.399 \mathrm{E}+05$ & 1.71 & 72.79 \\
\hline 1074 & $\mathrm{C}$ & $5.59 \mathrm{E}+02$ & 1.86001 & $5.2917 \mathrm{E}-08$ & 10.1422 & $1.21 \mathrm{E}-06$ & $1.383 \mathrm{E}+05$ & 1.49 & 72.15 \\
\hline 1075 & $\mathrm{C}$ & $5.34 \mathrm{E}+02$ & 1.83056 & 5.1882E-08 & 10.1459 & $1.17 \mathrm{E}-06$ & $1.368 \mathrm{E}+05$ & 1.28 & 71.52 \\
\hline 1076 & $\mathrm{C}$ & $5.10 \mathrm{E}+02$ & 1.80057 & $5.0800 \mathrm{E}-08$ & 10.1497 & $1.13 \mathrm{E}-06$ & $1.352 \mathrm{E}+05$ & 1.09 & 70.88 \\
\hline 1077 & $\mathrm{C}$ & $4.86 \mathrm{E}+02$ & 1.76619 & 4.9509E-08 & 10.1536 & $1.08 \mathrm{E}-06$ & $1.333 \mathrm{E}+05$ & 0.91 & 70.17 \\
\hline 1078 & $\mathrm{C}$ & $4.57 \mathrm{E}+02$ & 1.72789 & $4.7985 \mathrm{E}-08$ & 10.1586 & $1.03 \mathrm{E}-06$ & $1.312 \mathrm{E}+05$ & 0.73 & 69.36 \\
\hline 1079 & $\mathrm{C}$ & $4.29 \mathrm{E}+02$ & 1.69264 & $4.6470 \mathrm{E}-08$ & 10.1638 & $9.72 \mathrm{E}-07$ & $1.292 \mathrm{E}+05$ & 0.56 & 68.61 \\
\hline 1080 & $\mathrm{C}$ & $4.04 \mathrm{E}+02$ & 1.66094 & 4.4977E-08 & 10.1686 & $9.24 \mathrm{E}-07$ & $1.272 \mathrm{E}+05$ & 0.43 & 67.90 \\
\hline 1081 & $\mathrm{C}$ & $3.80 \mathrm{E}+02$ & 1.63122 & $4.3415 \mathrm{E}-08$ & 10.1735 & $8.75 \mathrm{E}-07$ & $1.252 \mathrm{E}+05$ & 0.32 & 67.18 \\
\hline 1082 & $\mathrm{C}$ & $3.56 \mathrm{E}+02$ & 1.60288 & $4.1784 \mathrm{E}-08$ & 10.1786 & $8.26 \mathrm{E}-07$ & $1.232 \mathrm{E}+05$ & 0.23 & 66.46 \\
\hline 1083 & $\mathrm{C}$ & $3.33 \mathrm{E}+02$ & 1.57488 & $4.0035 \mathrm{E}-08$ & 10.1838 & $7.78 \mathrm{E}-07$ & $1.210 \mathrm{E}+05$ & 0.16 & 65.72 \\
\hline 1084 & $\mathrm{C}$ & $3.09 \mathrm{E}+02$ & 1.54737 & $3.8160 \mathrm{E}-08$ & 10.1896 & $7.26 \mathrm{E}-07$ & $1.186 \mathrm{E}+05$ & 0.10 & 64.94 \\
\hline 1085 & $\mathrm{C}$ & $2.86 \mathrm{E}+02$ & 1.52243 & 3.6302E-08 & 10.1957 & $6.75 \mathrm{E}-07$ & $1.163 \mathrm{E}+05$ & 0.05 & 64.18 \\
\hline 1086 & $\mathrm{C}$ & $2.65 \mathrm{E}+02$ & 1.49992 & $3.4470 \mathrm{E}-08$ & 10.2015 & $6.29 \mathrm{E}-07$ & $1.140 \mathrm{E}+05$ & 0.01 & 63.44 \\
\hline 1087 & $\mathrm{R}$ & $2.45 \mathrm{E}+02$ & 1.48240 & $3.2925 \mathrm{E}-08$ & 10.2075 & $5.83 \mathrm{E}-07$ & $1.121 \mathrm{E}+05$ & 0.00 & 62.83 \\
\hline 1088 & $\mathrm{R}$ & $2.32 \mathrm{E}+02$ & 1.46981 & $3.1697 \mathrm{E}-08$ & 10.2115 & $5.54 \mathrm{E}-07$ & $1.105 \mathrm{E}+05$ & 0.00 & 62.35 \\
\hline 1089 & $\mathrm{R}$ & $2.19 \mathrm{E}+02$ & 1.45895 & 3.0510E-08 & 10.2156 & $5.24 \mathrm{E}-07$ & $1.090 \mathrm{E}+05$ & 0.00 & 61.88 \\
\hline 1090 & $\mathrm{R}$ & $2.08 \mathrm{E}+02$ & 1.44955 & 2.9369E-08 & 10.2194 & 4.99E-07 & $1.075 \mathrm{E}+05$ & 0.00 & 61.43 \\
\hline 1091 & $\mathrm{R}$ & $1.97 \mathrm{E}+02$ & 1.44085 & $2.8205 \mathrm{E}-08$ & 10.2234 & 4.73E-07 & $1.060 \mathrm{E}+05$ & 0.00 & 60.97 \\
\hline 1092 & $\mathrm{R}$ & $1.86 \mathrm{E}+02$ & 1.43173 & 2.6869E-08 & 10.2275 & $4.47 \mathrm{E}-07$ & $1.042 \mathrm{E}+05$ & 0.00 & 60.43 \\
\hline 1093 & $\mathrm{R}$ & $1.72 \mathrm{E}+02$ & 1.42204 & $2.5352 \mathrm{E}-08$ & 10.2329 & 4.15E-07 & $1.022 \mathrm{E}+05$ & 0.00 & 59.81 \\
\hline 1094 & $\mathrm{R}$ & $1.59 \mathrm{E}+02$ & 1.41419 & $2.4109 \mathrm{E}-08$ & 10.2385 & $3.82 \mathrm{E}-07$ & $1.005 \mathrm{E}+05$ & 0.00 & 59.29 \\
\hline 1095 & $\mathrm{R}$ & $1.50 \mathrm{E}+02$ & 1.40808 & $2.3160 \mathrm{E}-08$ & 10.2421 & $3.63 \mathrm{E}-07$ & $9.911 \mathrm{E}+04$ & 0.00 & 58.89 \\
\hline 1096 & $\mathrm{R}$ & $1.42 \mathrm{E}+02$ & 1.40244 & 2.2244E-08 & 10.2458 & 3.44E-07 & $9.779 \mathrm{E}+04$ & 0.00 & 58.50 \\
\hline 1097 & $\mathrm{R}$ & $1.35 \mathrm{E}+02$ & 1.39730 & $2.1365 \mathrm{E}-08$ & 10.2493 & 3.27E-07 & $9.650 \mathrm{E}+04$ & 0.00 & 58.12 \\
\hline 1098 & $\mathrm{R}$ & $1.28 \mathrm{E}+02$ & 1.39246 & $2.0485 \mathrm{E}-08$ & 10.2529 & $3.10 \mathrm{E}-07$ & $9.519 \mathrm{E}+04$ & 0.00 & 57.73 \\
\hline 1099 & $\mathrm{R}$ & $1.21 \mathrm{E}+02$ & 1.38794 & $1.9605 \mathrm{E}-08$ & 10.2565 & 2.93E-07 & $9.385 \mathrm{E}+04$ & 0.00 & 57.34 \\
\hline 1100 & $\mathrm{R}$ & $1.14 \mathrm{E}+02$ & 1.38372 & $1.8710 \mathrm{E}-08$ & 10.2603 & $2.76 \mathrm{E}-07$ & $9.246 \mathrm{E}+04$ & 0.00 & 56.93 \\
\hline 1101 & $\mathrm{R}$ & $1.08 \mathrm{E}+02$ & 1.37985 & $1.7798 \mathrm{E}-08$ & 10.2642 & 2.60E-07 & $9.101 \mathrm{E}+04$ & 0.00 & 56.51 \\
\hline 1102 & $\mathrm{R}$ & $1.01 \mathrm{E}+02$ & 1.37684 & $1.6995 \mathrm{E}-08$ & 10.2684 & $2.43 \mathrm{E}-07$ & $8.970 \mathrm{E}+04$ & 0.00 & 56.13 \\
\hline 1103 & $\mathrm{R}$ & $9.59 \mathrm{E}+01$ & 1.37462 & $1.6306 \mathrm{E}-08$ & 10.2716 & $2.31 \mathrm{E}-07$ & $8.855 \mathrm{E}+04$ & 0.00 & 55.80 \\
\hline 1104 & $\mathrm{R}$ & $9.10 \mathrm{E}+01$ & 1.37291 & $1.5607 \mathrm{E}-08$ & 10.2749 & 2.19E-07 & $8.736 \mathrm{E}+04$ & 0.00 & 55.46 \\
\hline 1105 & $\mathrm{R}$ & $8.61 \mathrm{E}+01$ & 1.37164 & $1.4811 \mathrm{E}-08$ & 10.2783 & 2.07E-07 & $8.597 \mathrm{E}+04$ & 0.00 & 55.07 \\
\hline 1106 & $\mathrm{R}$ & $8.01 \mathrm{E}+01$ & 1.37106 & $1.3914 \mathrm{E}-08$ & 10.2829 & $1.92 \mathrm{E}-07$ & $8.435 \mathrm{E}+04$ & 0.00 & 54.60 \\
\hline 1107 & $\mathrm{R}$ & $7.41 \mathrm{E}+01$ & 1.37119 & $1.3184 \mathrm{E}-08$ & 10.2877 & $1.77 \mathrm{E}-07$ & $8.299 \mathrm{E}+04$ & 0.00 & 54.22 \\
\hline 1108 & $\mathrm{R}$ & $7.04 \mathrm{E}+01$ & 1.37161 & $1.2631 \mathrm{E}-08$ & 10.2907 & $1.68 \mathrm{E}-07$ & $8.192 \mathrm{E}+04$ & 0.00 & 53.91 \\
\hline 1109 & $\mathrm{R}$ & $6.68 \mathrm{E}+01$ & 1.37234 & $1.2022 \mathrm{E}-08$ & 10.2938 & $1.59 \mathrm{E}-07$ & $8.072 \mathrm{E}+04$ & 0.00 & 53.57 \\
\hline 1110 & $\mathrm{R}$ & $6.26 \mathrm{E}+01$ & 1.37335 & $1.1354 \mathrm{E}-08$ & 10.2977 & $1.48 \mathrm{E}-07$ & $7.936 \mathrm{E}+04$ & 0.00 & 53.18 \\
\hline 1111 & $\mathrm{R}$ & $5.83 \mathrm{E}+01$ & 1.37444 & $1.0789 \mathrm{E}-08$ & 10.3018 & $1.37 \mathrm{E}-07$ & $7.817 \mathrm{E}+04$ & 0.00 & 52.85 \\
\hline 1112 & $\mathrm{R}$ & $5.55 \mathrm{E}+01$ & 1.37559 & $1.0338 \mathrm{E}-08$ & 10.3047 & $1.30 \mathrm{E}-07$ & $7.720 \mathrm{E}+04$ & 0.00 & 52.57 \\
\hline 1113 & $\mathrm{R}$ & $5.27 \mathrm{E}+01$ & 1.37700 & 9.8872E-09 & 10.3076 & $1.24 \mathrm{E}-07$ & $7.620 \mathrm{E}+04$ & 0.00 & 52.29 \\
\hline 1114 & $\mathrm{R}$ & $5.00 \mathrm{E}+01$ & 1.37874 & $9.4310 \mathrm{E}-09$ & 10.3107 & $1.17 \mathrm{E}-07$ & $7.516 \mathrm{E}+04$ & 0.00 & 51.99 \\
\hline 1115 & $\mathrm{R}$ & $4.72 \mathrm{E}+01$ & 1.38088 & 8.9689E-09 & 10.3139 & $1.10 \mathrm{E}-07$ & $7.407 \mathrm{E}+04$ & 0.00 & 51.69 \\
\hline 1116 & $\mathrm{R}$ & $4.44 \mathrm{E}+01$ & 1.38319 & $8.5480 \mathrm{E}-09$ & 10.3173 & $1.03 \mathrm{E}-07$ & $7.305 \mathrm{E}+04$ & 0.00 & 51.40 \\
\hline 1117 & $\mathrm{R}$ & $4.22 \mathrm{E}+01$ & 1.38562 & $8.1697 \mathrm{E}-09$ & 10.3201 & $9.76 \mathrm{E}-08$ & $7.211 \mathrm{E}+04$ & 0.00 & 51.13 \\
\hline 1118 & $\mathrm{R}$ & $4.00 \mathrm{E}+01$ & 1.38845 & 7.7869E-09 & 10.3230 & 9.22E-08 & $7.112 \mathrm{E}+04$ & 0.00 & 50.85 \\
\hline 1119 & $\mathrm{R}$ & $3.78 \mathrm{E}+01$ & 1.39177 & 7.3995E-09 & 10.3261 & 8.67E-08 & $7.010 \mathrm{E}+04$ & 0.00 & 50.56 \\
\hline 1120 & $\mathrm{R}$ & $3.56 \mathrm{E}+01$ & 1.39617 & $6.9626 \mathrm{E}-09$ & 10.3293 & $8.12 \mathrm{E}-08$ & $6.890 \mathrm{E}+04$ & 0.00 & 50.23 \\
\hline 1121 & $\mathrm{R}$ & $3.29 \mathrm{E}+01$ & 1.40204 & $6.4740 \mathrm{E}-09$ & 10.3336 & 7.45E-08 & $6.750 \mathrm{E}+04$ & 0.00 & 49.83 \\
\hline 1122 & $\mathrm{R}$ & $3.02 \mathrm{E}+01$ & 1.40739 & $6.0948 \mathrm{E}-09$ & 10.3381 & $6.79 \mathrm{E}-08$ & $6.636 \mathrm{E}+04$ & 0.00 & 49.51 \\
\hline 1123 & $\mathrm{R}$ & $2.88 \mathrm{E}+01$ & 1.41162 & $5.8291 \mathrm{E}-09$ & 10.3406 & 6.43E-08 & $6.554 \mathrm{E}+04$ & 0.00 & 49.27 \\
\hline 1124 & $\mathrm{R}$ & $2.73 \mathrm{E}+01$ & 1.41634 & $5.5609 \mathrm{E}-09$ & 10.3433 & $6.08 \mathrm{E}-08$ & $6.468 \mathrm{E}+04$ & 0.00 & 49.03 \\
\hline 1125 & $\mathrm{R}$ & $2.59 \mathrm{E}+01$ & 1.42226 & $5.2595 \mathrm{E}-09$ & 10.3461 & $5.72 \mathrm{E}-08$ & $6.368 \mathrm{E}+04$ & 0.00 & 48.74 \\
\hline 1126 & $\mathrm{R}$ & $2.41 \mathrm{E}+01$ & 1.42985 & 4.9241E-09 & 10.3497 & $5.29 \mathrm{E}-08$ & $6.252 \mathrm{E}+04$ & 0.00 & 48.41 \\
\hline 1127 & $\mathrm{R}$ & $2.23 \mathrm{E}+01$ & 1.43837 & 4.6133E-09 & 10.3536 & 4.86E-08 & $6.140 \mathrm{E}+04$ & 0.00 & 48.09 \\
\hline 1128 & $\mathrm{R}$ & $2.08 \mathrm{E}+01$ & 1.44656 & 4.3587E-09 & 10.3570 & $4.50 \mathrm{E}-08$ & $6.043 \mathrm{E}+04$ & 0.00 & 47.81 \\
\hline 1129 & $\mathrm{R}$ & $1.96 \mathrm{E}+01$ & 1.45480 & 4.1322E-09 & 10.3598 & 4.22E-08 & $5.954 \mathrm{E}+04$ & 0.00 & 47.54 \\
\hline 1130 & $\mathrm{R}$ & $1.84 \mathrm{E}+01$ & 1.46274 & 3.9346E-09 & 10.3628 & 3.93E-08 & $5.873 \mathrm{E}+04$ & 0.00 & 47.30 \\
\hline 1131 & $\mathrm{R}$ & $1.76 \mathrm{E}+01$ & 1.47005 & $3.7666 \mathrm{E}-09$ & 10.3651 & $3.73 \mathrm{E}-08$ & $5.802 \mathrm{E}+04$ & 0.00 & 47.08 \\
\hline 1132 & $\mathrm{R}$ & $1.67 \mathrm{E}+01$ & 1.47948 & 3.5652E-09 & 10.3675 & 3.52E-08 & $5.712 \mathrm{E}+04$ & 0.00 & 46.80 \\
\hline 1133 & $\mathrm{R}$ & $1.55 \mathrm{E}+01$ & 1.48989 & 3.3581E-09 & 10.3710 & 3.24E-08 & $5.617 \mathrm{E}+04$ & 0.00 & 46.50 \\
\hline 1134 & $\mathrm{R}$ & $1.45 \mathrm{E}+01$ & 1.50018 & $3.1779 \mathrm{E}-09$ & 10.3738 & $3.02 \mathrm{E}-08$ & $5.529 \mathrm{E}+04$ & 0.00 & 46.22 \\
\hline 1135 & $\mathrm{R}$ & $1.36 \mathrm{E}+01$ & 1.51024 & $3.0255 \mathrm{E}-09$ & 10.3767 & $2.80 \mathrm{E}-08$ & $5.452 \mathrm{E}+04$ & 0.00 & 45.96 \\
\hline 1136 & $\mathrm{R}$ & $1.29 \mathrm{E}+01$ & 1.51938 & 2.9012E-09 & 10.3789 & $2.66 \mathrm{E}-08$ & $5.386 \mathrm{E}+04$ & 0.00 & 45.74 \\
\hline 1137 & $\mathrm{R}$ & $1.23 \mathrm{E}+01$ & 1.52942 & $2.7765 \mathrm{E}-09$ & 10.3811 & $2.51 \mathrm{E}-08$ & $5.318 \mathrm{E}+04$ & 0.00 & 45.51 \\
\hline
\end{tabular}


M. Cantiello et al.: Sub-surface convection in hot stars, Online Material $p 7$

Table A.2. continued.

\begin{tabular}{|c|c|c|c|c|c|c|c|c|c|}
\hline Grid point & Stat & $\tau$ & $x$ & $\rho$ & 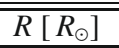 & $\overline{M_{*}-M_{r}[\mathrm{~g}]}$ & $\bar{T} \overline{T[\mathrm{~K}]}$ & $\overline{v_{\mathrm{c}}\left[\mathrm{km} \mathrm{s}^{-1}\right]}$ & $\bar{c}_{c_{\mathrm{s}}\left[\mathrm{km} \mathrm{s}^{-1}\right]}$ \\
\hline 1138 & $\mathrm{R}$ & $1.16 \mathrm{E}+01$ & 1.54073 & $2.6471 \mathrm{E}-09$ & 10.3834 & $2.36 \mathrm{E}-08$ & $5.244 \mathrm{E}+04$ & 0.00 & 45.24 \\
\hline 1139 & $\mathrm{R}$ & $1.09 \mathrm{E}+01$ & 1.55335 & $2.5130 \mathrm{E}-09$ & 10.3860 & $2.20 \mathrm{E}-08$ & $5.164 \mathrm{E}+04$ & 0.00 & 44.94 \\
\hline 1140 & $\mathrm{R}$ & $1.02 \mathrm{E}+01$ & 1.56677 & $2.3784 \mathrm{E}-09$ & 10.3887 & $2.05 \mathrm{E}-08$ & $5.079 \mathrm{E}+04$ & 0.00 & 44.61 \\
\hline 1141 & $\mathrm{R}$ & $9.50 \mathrm{E}+00$ & 1.58081 & 2.2433E-09 & 10.3916 & 1.89E-08 & $4.988 \mathrm{E}+04$ & 0.00 & 44.25 \\
\hline 1142 & $\mathrm{R}$ & $8.78 \mathrm{E}+00$ & 1.59453 & 2.1289E-09 & 10.3946 & $1.73 \mathrm{E}-08$ & $4.908 \mathrm{E}+04$ & 0.00 & 43.91 \\
\hline 1143 & $\mathrm{R}$ & $8.28 \mathrm{E}+00$ & 1.60744 & $2.0356 \mathrm{E}-09$ & 10.3968 & $1.62 \mathrm{E}-08$ & $4.838 \mathrm{E}+04$ & 0.00 & 43.60 \\
\hline 1144 & $\mathrm{R}$ & $7.77 \mathrm{E}+00$ & 1.62179 & $1.9422 \mathrm{E}-09$ & 10.3991 & $1.51 \mathrm{E}-08$ & $4.765 \mathrm{E}+04$ & 0.00 & 43.25 \\
\hline 1145 & $\mathrm{R}$ & $7.26 \mathrm{E}+00$ & 1.63733 & 1.8489E-09 & 10.4016 & $1.41 \mathrm{E}-08$ & $4.688 \mathrm{E}+04$ & 0.00 & 42.87 \\
\hline 1146 & $\mathrm{R}$ & $6.75 \mathrm{E}+00$ & 1.65130 & $1.7685 \mathrm{E}-09$ & 10.4041 & $1.30 \mathrm{E}-08$ & $4.618 \mathrm{E}+04$ & 0.00 & 42.50 \\
\hline 1147 & $\mathrm{R}$ & $6.37 \mathrm{E}+00$ & 1.66300 & $1.7008 \mathrm{E}-09$ & 10.4060 & $1.22 \mathrm{E}-08$ & $4.555 \mathrm{E}+04$ & 0.00 & 42.15 \\
\hline 1148 & $\mathrm{R}$ & $5.99 \mathrm{E}+00$ & 1.67593 & $1.6205 \mathrm{E}-09$ & 10.4080 & 1.14E-08 & $4.477 \mathrm{E}+04$ & 0.00 & 41.69 \\
\hline 1149 & $\mathrm{C}$ & $5.46 \mathrm{E}+00$ & 1.68633 & $1.5492 \mathrm{E}-09$ & 10.4109 & 1.03E-08 & $4.404 \mathrm{E}+04$ & 0.07 & 41.23 \\
\hline 1150 & $\mathrm{C}$ & $5.18 \mathrm{E}+00$ & 1.69348 & $1.4995 \mathrm{E}-09$ & 10.4125 & $9.75 \mathrm{E}-09$ & $4.350 \mathrm{E}+04$ & 0.07 & 40.87 \\
\hline 1151 & $\mathrm{C}$ & $4.89 \mathrm{E}+00$ & 1.70032 & $1.4498 \mathrm{E}-09$ & 10.4142 & $9.17 \mathrm{E}-09$ & $4.294 \mathrm{E}+04$ & 0.07 & 40.47 \\
\hline 1152 & $\mathrm{C}$ & $4.61 \mathrm{E}+00$ & 1.70654 & $1.4000 \mathrm{E}-09$ & 10.4159 & 8.59E-09 & $4.236 \mathrm{E}+04$ & 0.08 & 40.03 \\
\hline 1153 & $\mathrm{C}$ & $4.32 \mathrm{E}+00$ & 1.71194 & $1.3475 \mathrm{E}-09$ & 10.4177 & 8.02E-09 & $4.171 \mathrm{E}+04$ & 0.08 & 39.52 \\
\hline 1154 & $\mathrm{C}$ & $4.00 \mathrm{E}+00$ & 1.71555 & $1.2921 \mathrm{E}-09$ & 10.4198 & 7.38E-09 & $4.100 \mathrm{E}+04$ & 0.08 & 38.92 \\
\hline 1155 & $\mathrm{C}$ & $3.68 \mathrm{E}+00$ & 1.71615 & $1.2488 \mathrm{E}-09$ & 10.4219 & $6.74 \mathrm{E}-09$ & $4.042 \mathrm{E}+04$ & 0.09 & 38.41 \\
\hline 1156 & $\mathrm{C}$ & $3.51 \mathrm{E}+00$ & 1.71368 & $1.2020 \mathrm{E}-09$ & 10.4231 & $6.38 \mathrm{E}-09$ & $3.977 \mathrm{E}+04$ & 0.09 & 37.80 \\
\hline 1157 & $\mathrm{C}$ & $3.16 \mathrm{E}+00$ & 1.70637 & $1.1546 \mathrm{E}-09$ & 10.4257 & 5.67E-09 & $3.909 \mathrm{E}+04$ & 0.09 & 37.14 \\
\hline 1158 & $\mathrm{C}$ & $2.98 \mathrm{E}+00$ & 1.69773 & $1.1208 \mathrm{E}-09$ & 10.4270 & 5.31E-09 & $3.859 \mathrm{E}+04$ & 0.10 & 36.66 \\
\hline 1159 & $\mathrm{C}$ & $2.79 \mathrm{E}+00$ & 1.68575 & $1.0858 \mathrm{E}-09$ & 10.4285 & 4.92E-09 & $3.806 \mathrm{E}+04$ & 0.09 & 36.16 \\
\hline 1160 & $\mathrm{C}$ & $2.61 \mathrm{E}+00$ & 1.67097 & $1.0512 \mathrm{E}-09$ & 10.4300 & $4.55 \mathrm{E}-09$ & $3.754 \mathrm{E}+04$ & 0.13 & 35.67 \\
\hline 1161 & $\mathrm{C}$ & $2.43 \mathrm{E}+00$ & 1.65364 & $1.0168 \mathrm{E}-09$ & 10.4315 & $4.18 \mathrm{E}-09$ & $3.701 \mathrm{E}+04$ & 0.12 & 35.21 \\
\hline 1162 & $\mathrm{C}$ & $2.27 \mathrm{E}+00$ & 1.63409 & $9.8245 \mathrm{E}-10$ & 10.4330 & 3.84E-09 & $3.649 \mathrm{E}+04$ & 0.11 & 34.79 \\
\hline 1163 & $\mathrm{C}$ & $2.11 \mathrm{E}+00$ & 1.61186 & $9.4679 \mathrm{E}-10$ & 10.4345 & 3.49E-09 & $3.596 \mathrm{E}+04$ & 0.13 & 34.41 \\
\hline 1164 & $\mathrm{C}$ & $1.95 \mathrm{E}+00$ & 1.59444 & $9.2036 \mathrm{E}-10$ & 10.4361 & $3.15 \mathrm{E}-09$ & $3.556 \mathrm{E}+04$ & 0.14 & 34.16 \\
\hline 1165 & $\mathrm{C}$ & $1.88 \mathrm{E}+00$ & 1.58330 & $9.0408 \mathrm{E}-10$ & 10.4367 & $3.00 \mathrm{E}-09$ & $3.532 \mathrm{E}+04$ & 0.16 & 34.02 \\
\hline 1166 & $\mathrm{C}$ & $1.81 \mathrm{E}+00$ & 1.56475 & $8.7896 \mathrm{E}-10$ & 10.4374 & $2.85 \mathrm{E}-09$ & $3.496 \mathrm{E}+04$ & 0.16 & 33.84 \\
\hline 1167 & $\mathrm{C}$ & $1.68 \mathrm{E}+00$ & 1.54394 & $8.5291 \mathrm{E}-10$ & 10.4389 & $2.56 \mathrm{E}-09$ & $3.458 \mathrm{E}+04$ & 0.16 & 33.68 \\
\hline 1168 & $\mathrm{C}$ & $1.61 \mathrm{E}+00$ & 1.52883 & $8.3498 \mathrm{E}-10$ & 10.4396 & 2.41E-09 & $3.433 \mathrm{E}+04$ & 0.17 & 33.59 \\
\hline 1169 & $\mathrm{C}$ & $1.55 \mathrm{E}+00$ & 1.51274 & $8.1655 \mathrm{E}-10$ & 10.4404 & 2.26E-09 & $3.408 \mathrm{E}+04$ & 0.19 & 33.52 \\
\hline 1170 & $\mathrm{C}$ & $1.48 \mathrm{E}+00$ & 1.48977 & 7.9113E-10 & 10.4412 & $2.11 \mathrm{E}-09$ & $3.373 \mathrm{E}+04$ & 0.19 & 33.43 \\
\hline 1171 & $\mathrm{R}$ & $1.38 \mathrm{E}+00$ & 1.46832 & $7.6805 \mathrm{E}-10$ & 10.4425 & $1.87 \mathrm{E}-09$ & $3.342 \mathrm{E}+04$ & 0.00 & 33.38 \\
\hline 1172 & $\mathrm{R}$ & $1.34 \mathrm{E}+00$ & 1.44895 & $7.4758 \mathrm{E}-10$ & 10.4431 & $1.77 \mathrm{E}-09$ & $3.316 \mathrm{E}+04$ & 0.00 & 33.34 \\
\hline 1173 & $\mathrm{R}$ & $1.25 \mathrm{E}+00$ & 1.42850 & $7.2624 \mathrm{E}-10$ & 10.4442 & $1.57 \mathrm{E}-09$ & $3.289 \mathrm{E}+04$ & 0.00 & 33.32 \\
\hline 1174 & $\mathrm{R}$ & $1.21 \mathrm{E}+00$ & 1.41409 & $7.1132 \mathrm{E}-10$ & 10.4448 & $1.47 \mathrm{E}-09$ & $3.270 \mathrm{E}+04$ & 0.00 & 33.31 \\
\hline 1175 & $\mathrm{R}$ & $1.17 \mathrm{E}+00$ & 1.39428 & $6.9089 \mathrm{E}-10$ & 10.4454 & 1.37E-09 & $3.245 \mathrm{E}+04$ & 0.00 & 33.31 \\
\hline 1176 & $\mathrm{R}$ & $1.10 \mathrm{E}+00$ & 1.36990 & $6.6582 \mathrm{E}-10$ & 10.4465 & 1.20E-09 & $3.215 \mathrm{E}+04$ & 0.00 & 33.32 \\
\hline 1177 & $\mathrm{R}$ & $1.05 \mathrm{E}+00$ & 1.34597 & $6.4121 \mathrm{E}-10$ & 10.4474 & $1.05 \mathrm{E}-09$ & $3.187 \mathrm{E}+04$ & 0.00 & 33.35 \\
\hline 1178 & $\mathrm{R}$ & $9.87 \mathrm{E}-01$ & 1.32442 & $6.1900 \mathrm{E}-10$ & 10.4485 & $8.97 \mathrm{E}-10$ & $3.162 \mathrm{E}+04$ & 0.00 & 33.38 \\
\hline 1179 & $\mathrm{R}$ & $9.44 \mathrm{E}-01$ & 1.30661 & $6.0086 \mathrm{E}-10$ & 10.4493 & $7.84 \mathrm{E}-10$ & $3.141 \mathrm{E}+04$ & 0.00 & 33.42 \\
\hline 1180 & $\mathrm{R}$ & $9.07 \mathrm{E}-01$ & 1.28575 & $5.8007 \mathrm{E}-10$ & 10.4500 & $6.86 \mathrm{E}-10$ & $3.119 \mathrm{E}+04$ & 0.00 & 33.47 \\
\hline 1181 & $\mathrm{R}$ & $8.57 \mathrm{E}-01$ & 1.26672 & $5.6146 \mathrm{E}-10$ & 10.4510 & $5.48 \mathrm{E}-10$ & $3.099 \mathrm{E}+04$ & 0.00 & 33.52 \\
\hline 1182 & $\mathrm{R}$ & 8.32E-01 & 1.25106 & $5.4637 \mathrm{E}-10$ & 10.4515 & $4.81 \mathrm{E}-10$ & $3.083 \mathrm{E}+04$ & 0.00 & 33.57 \\
\hline 1183 & $\mathrm{R}$ & 7.98E-01 & 1.23145 & $5.2773 \mathrm{E}-10$ & 10.4522 & $3.85 \mathrm{E}-10$ & $3.064 \mathrm{E}+04$ & 0.00 & 33.64 \\
\hline 1184 & $\mathrm{R}$ & 7.63E-01 & 1.20815 & $5.0587 \mathrm{E}-10$ & 10.4531 & $2.84 \mathrm{E}-10$ & $3.042 \mathrm{E}+04$ & 0.00 & 33.74 \\
\hline 1185 & $\mathrm{R}$ & $7.21 \mathrm{E}-01$ & 1.18955 & $4.8863 \mathrm{E}-10$ & 10.4541 & $1.60 \mathrm{E}-10$ & $3.025 \mathrm{E}+04$ & 0.00 & 33.84 \\
\hline 1186 & $\mathrm{R}$ & 7.04E-01 & 1.17182 & $4.7235 \mathrm{E}-10$ & 10.4545 & $1.11 \mathrm{E}-10$ & $3.010 \mathrm{E}+04$ & 0.00 & 33.93 \\
\hline 1187 & $\mathrm{R}$ & 6.67E-01 & 1.15923 & $4.6089 \mathrm{E}-10$ & 10.4556 & $0.00 \mathrm{E}+00$ & $2.999 \mathrm{E}+04$ & 0.00 & 34.01 \\
\hline
\end{tabular}

